\title{
Supersymmetry of AdS and flat backgrounds in M-theory
}

\section{J. Gutowski ${ }^{a}$ and G. Papadopoulos ${ }^{b}$}

${ }^{a}$ Department of Mathematics, University of Surrey, Guildford, GU2 7XH, U.K.

${ }^{b}$ Department of Mathematics, King's College London, Strand, London WC2R 2LS, U.K.

E-mail: j.gutowski@surrey.ac.uk, george.papadopoulos@kcl.ac.uk

ABSTRACT: We give a systematic description of all warped $A d S_{n}$ and $\mathbb{R}^{n-1,1}$ backgrounds of M-theory and identify the a priori number of supersymmetries that these backgrounds preserve. In particular, we show that $A d S_{n}$ backgrounds preserve $N=2^{\left[\frac{n}{2}\right]} k$ for $n \leq 4$ and $N=2^{\left[\frac{n}{2}\right]+1} k$ for $4<n \leq 7$ supersymmetries while $\mathbb{R}^{n-1,1}$ backgrounds preserve $N=2^{\left[\frac{n}{2}\right]} k$ for $n \leq 4$ and $N=2^{\left[\frac{n+1}{2}\right]} k$ for $4<n \leq 7$, supersymmetries. Furthermore for $A d S_{n}$ backgrounds that satisfy the requirements for the maximum principle to hold, we show that the Killing spinors can be identified with the zero modes of Dirac-like operators on $M^{11-n}$ coupled to fluxes thus establishing a new class of Lichnerowicz type theorems. We also demonstrate that the Killing spinors of generic warped $A d S_{n}$ backgrounds do not factorize into products of Killing spinors on $A d S_{n}$ and Killing spinors on the transverse space.

KEYwords: Black Holes in String Theory, Supergravity Models

ARXIV EPRINT: 1407.5652 


\section{Contents}

1 Introduction 1

2 AdS backgrounds and near horizon geometries 5

2.1 Warped AdS and flat backgrounds 5

$\begin{array}{lll}2.1 .1 & A d S_{2} \times{ }_{w} M^{9} & 6\end{array}$

$\begin{array}{lll}2.1 .2 & A d S_{3} \times{ }_{w} M^{8} & 6\end{array}$

$\begin{array}{lll}2.1 .3 & A d S_{4} \times_{w} M^{7} & 6\end{array}$

2.1.4 $A d S_{n} \times{ }_{w} M^{11-n}, n>4 \quad 6$

2.1.5 $\mathbb{R}^{n-1,1} \times_{w} M^{11-n} \quad 7$

$\begin{array}{lll}2.2 & \text { Bianchi identities and field equations } & 7\end{array}$

2.3 Killing spinor equations 8

2.3.1 Light-cone integrability and independent KSEs 9

$\begin{array}{lll}3 & \boldsymbol{A d} \boldsymbol{S}_{2} \text { : local analysis } & 10\end{array}$

$\begin{array}{lll}3.1 \text { Field equations } & 10\end{array}$

$\begin{array}{ll}\text { 3.1.1 The warp factor } A \text { is nowhere vanishing } & 10\end{array}$

$\begin{array}{lll}3.2 & \text { Killing spinor equations } & 11\end{array}$

$4 \boldsymbol{A d S}_{2}$ : global analysis $\quad 11$

4.1 A Lichnerowicz type of theorem for $\mathcal{D}^{(+)} \quad 11$

$\begin{array}{lll}4.2 & \text { A Lichnerowicz theorem for } \mathcal{D}^{(-)} & 12\end{array}$

$\begin{array}{lll}4.3 & \text { Counting supersymmetries } & 13\end{array}$

$5 \mathrm{AdS}_{3}$ : local Analysis $\quad 13$

$\begin{array}{ll}5.1 \text { Bianchi identities and field equations } & 13\end{array}$

$\begin{array}{ll}\text { 5.1.1 The warp factor } A \text { is nowhere vanishing } & 14\end{array}$

$\begin{array}{lll}5.2 & \text { Killing spinor equations } & 14\end{array}$

$\begin{array}{ll}5.3 \text { Counting supersymmetries } & 16\end{array}$

$6 \quad \boldsymbol{A d} S_{3}$ : global Analysis $\quad 16$

6.1 A new Lichnerowicz theorem for $\tau_{+}$and $\sigma_{+} \quad 16$

$\begin{array}{ll}\text { 6.2 A new Lichnerowicz theorem for } \tau_{-} \text {and } \sigma_{-} & 19\end{array}$

$\begin{array}{lll}6.3 & \text { Counting supersymmetries again } 20\end{array}$

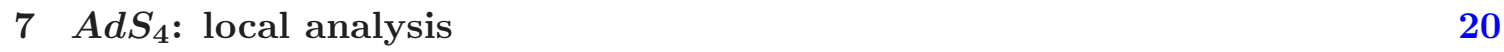

$\begin{array}{ll}7.1 \text { Field equations } & 20\end{array}$

$\begin{array}{ll}\text { 7.1.1 The warp factor } A \text { is nowhere vanishing } & 21\end{array}$

$\begin{array}{lll}7.2 & \text { Integrability of KSEs along AdS } & 21\end{array}$

$\begin{array}{lll}7.3 & \text { Counting supersymmetries } & 22\end{array}$ 
$8 \quad \mathrm{AdS}_{4}$ : global analysis $\quad \mathbf{2 2}$

8.1 A new Lichnerowicz theorem for $\tau_{+}$and $\sigma_{+} \quad 22$

8.2 A new Lichnerowicz theorem for $\tau_{-}$and $\sigma_{-} \quad 23$

$\begin{array}{ll}8.3 \text { Counting supersymmetries } & 23\end{array}$

$9 \operatorname{AdS}_{n} n>4$ : local analysis $\quad 24$

$\begin{array}{lll}9.1 & \text { Bianchi identities and field equations } & 24\end{array}$

$\begin{array}{lll}\text { 9.1.1 The warp factor } A \text { is nowhere vanishing } & 24\end{array}$

9.2 Integrability of KSEs along AdS 24

$\begin{array}{lll}9.3 & \text { Counting supersymmetries } & 25\end{array}$

$10 \operatorname{AdS}_{n} n>4$ : global analysis $\quad 26$

10.1 A new Lichnerowicz Theorem for $\tau_{+}$and $\sigma_{+} \quad 26$

10.2 A new Lichnerowicz Theorem for $\tau_{-}$and $\sigma_{-} \quad 27$

10.3 Counting supersymmetries 28

11 Do the Killing spinors factorize? 28

$12 \mathbb{R}^{n-1,1}$ M-theory backgrounds $\quad 31$

12.1 The warp factor is not nowhere vanishing 31

12.2 Counting supersymmetries 31

$\begin{array}{ll}12.2 .1 \mathbb{R}^{1,1} \text { backgrounds } & 32\end{array}$

$\begin{array}{ll}12.2 .2 \mathbb{R}^{2,1} \text { backgrounds } & 32\end{array}$

$\begin{array}{ll}12.2 .3 \mathbb{R}^{3,1} \text { backgrounds } & 33\end{array}$

12.2.4 $\mathbb{R}^{n-1,1}, n>4$ backgrounds 33

13 Concluding remarks $\quad 34$

$\begin{array}{ll}\text { A Notation and conventions } & 35\end{array}$

\section{Introduction}

The AdS backgrounds in eleven dimensions, following the original work of Freund and Rubin [1], have found widespread applications either as supergravity compactifications, see $[2,3]$ for reviews, or more recently in the AdS/CFT correspondence as it applies to M-theory [4]. Because of this, there is an extensive literature in the construction of AdS backgrounds with emphasis on those preserving some of the supersymmetry of the underlying supergravity theory, for some selected references that include applications see [5]-[14]. Most of the computations made so far apply to special cases. These involve either restrictions on the number of active fields or a priori assumptions on the form of Killing spinors.

The purpose of this paper is to give a comprehensive description of all warped AdS and flat backgrounds of 11-dimensional supergravity, $A d S_{n} \times{ }_{w} M^{11-n}$ and $\mathbb{R}^{n-1,1} \times{ }_{w} M^{11-n}$, 
respectively, making use of at most two assumptions. ${ }^{1}$ One assumption is that the backgrounds admit at least one supersymmetry. This applies to all cases that will be described in this paper. The second assumption, which applies to only parts of the analysis, is that the fields and $M^{11-n}$ satisfy the requirements necessary for the maximum principle to apply. Such requirements include the assumptions that the fields are smooth and that $M^{11-n}$ is compact without boundary.

The focus of this paper is to count the number supersymmetries preserved by $A d S_{n}$ and $\mathbb{R}^{n-1,1}$ backgrounds. In another paper [15], we shall describe the restrictions on the geometry of $M^{11-n}$ imposed by the KSEs and field equations of 11-dimensional supergravity. We have also included a brief description of the geometry of some of the $A d S_{n}$ backgrounds.

Our results include the identification of all a priori fractions of supersymmetry preserved by the $A d S_{n} \times{ }_{w} M^{11-n}$ and $\mathbb{R}^{n-1,1} \times{ }_{w} M^{11-n}$ backgrounds. In particular assuming that such backgrounds exist, we count the number of Killing spinors $N$ that they preserve. We find that for $A d S_{n}$ backgrounds

$$
N=2^{\left[\frac{n}{2}\right]} k, \quad n \leq 4 ; \quad N=2^{\left[\frac{n}{2}\right]+1} k, \quad 4<n \leq 7
$$

where $k \in \mathbb{N}_{>0}$. To prove this result for $A d S_{2}$ backgrounds we have assumed that the fields and $M^{9}$ satisfy the requirements of the maximum principle. However for the rest of the $A d S_{n}$ backgrounds, this assumption is not necessary and the counting of supersymmetries stated is valid more generally. The number of supersymmetries $N$ is further restricted. For example, restrictions arise from the classification results of [16-18] on backgrounds with maximal and near maximal supersymmetries. In addition all backgrounds preserving more than 16 supersymmetries are homogeneous [19]. The results have been tabulated in table 1.

The number of supersymmetries preserved by $A d S_{n}$ backgrounds can also be determined by counting the zero modes of appropriate Dirac-like operators $\mathscr{D}^{( \pm)}$coupled to fluxes on the transverse spaces $M^{11-n}$. This is achieved by proving new types of Lichnerowicz theorems which give a 1-1 correspondence between the Killing spinors and the zero modes of $\mathscr{D}^{( \pm)}$. To prove these theorems, we assume that the fields and $M^{11-n}$ satisfy all the conditions required for the maximum principle to hold. As a result, we show that the number of supersymmetries of $A d S_{n}$ backgrounds can be rewritten as

$$
N=\ell(n) N_{-},
$$

where $\ell(n)=2^{\left[\frac{n}{2}\right]}, 2 \leq n \leq 4$ and $\ell(n)=4,4<n \leq 7$ and $N_{-}=\operatorname{dim} \operatorname{Ker} \mathscr{D}^{(-)}$.

The explicit expression we obtain for the Killing spinors of $A d S_{n}$ backgrounds allows us to investigate whether they can be factorized into the form $\epsilon=\xi \otimes \psi$, where $\xi$ is a Killing spinor on $A d S_{n}$ and $\psi$ is a Killing spinor on $M^{11-n}$. Such a factorization has been widely used in the literature to solve the KSEs for such backgrounds. We find that for this factorization to hold an additional condition must be imposed on the Killing spinors which does not arise from the solution of the KSEs on the spacetime. Because of this we conclude that such a factorization does not hold for generic backgrounds. We demonstrate

\footnotetext{
${ }^{1}$ It is also assumed that the fields are sufficiently differentiable.
} 
with an explicit example that for the correct counting of supersymmetries, one should not assume the above factorization.

Our $A d S_{n}$ results can be adapted to $\mathbb{R}^{n-1,1}$ or equivalently flat backgrounds. The latter arise in the limit where the AdS radius goes to infinity. This limit can be taken smoothly in all our local computations. But some of the regularity properties enjoyed by the $A d S_{n}$ backgrounds do not extend in the limit. One of the main consequences of this is that the new Lichnerowicz theorems we have established for $A d S_{n}$ do not hold for flat backgrounds. As a result, one cannot prove that $\mathbb{R}^{1,1}$ backgrounds always preserve an even number of supersymmetries. However for the rest of the $\mathbb{R}^{n-1,1}$ backgrounds, we shall show that

$$
N=2^{\left[\frac{n}{2}\right]} k, \quad 2<n \leq 4 ; \quad N=2^{\left[\frac{n+1}{2}\right]} k, \quad 4<n<8 .
$$

$N$ is further restricted. Observe that the number of supersymmetries preserved by the $A d S_{n}$ and $\mathbb{R}^{n-1,1}$ backgrounds differs. This is because the counting of linearly independent Killing spinors is different in the two cases.

The method we use to solve the KSEs for each of the $A d S_{n} \times{ }_{w} M^{11-n}$ backgrounds is based on the observation of [20] that all such backgrounds can be described as near horizon geometries, and that the KSEs can be integrated for M-horizons [21-23]. In particular, we use this to integrate the gravitino KSE of $\mathrm{D}=11$ supergravity along all $A d S_{n}$ directions and then identify the independent KSEs on $M^{11-n}$. The integration of KSEs along $A d S_{n}$ first involves the integration along two lightcone directions which arise naturally in the description of $A d S_{n}$ backgrounds as near horizon geometries. This is achieved after decomposing the Killing spinor as $\epsilon=\epsilon_{+}+\epsilon_{-}$according to some lightcone projections $\Gamma_{ \pm} \epsilon_{ \pm}=0$. This integration allows the Killing spinors to be written as $\epsilon_{ \pm}=\epsilon_{ \pm}\left(r, u, \phi_{ \pm}\right)$, where $\phi_{ \pm}$depend only on the coordinates of the co-dimension two subspace $\mathcal{S}$ defined by $r=u=0$. A key computation described in [22, 23], after using Bianchi identities and field equations, reveals that there are two remaining independent KSEs one for each $\phi_{ \pm}$, which are derived from the naive restriction of the KSE equation of $\mathrm{D}=11$ supergravity on $\mathcal{S}$. This is sufficient to establish the formulae (1.1) and (1.2) for $A d S_{2}$ backgrounds. This is because such backgrounds are special cases of horizons and so these formulae already follow from the results of [21-23]. For $A d S_{n}, n>2$, to derive (1.1), it is necessary to integrate along the rest of the $A d S_{n}$ directions. It turns out that this is always possible at the cost of introducing additional algebraic KSEs on $M^{11-n}$. The Killing spinors can be expressed as $\phi_{+}=\phi_{+}\left(x, \tau_{+}, \sigma_{+}\right)$and $\phi_{-}=\phi_{-}\left(x, \tau_{-}, \sigma_{-}\right)$, where $x$ denote collectively the remaining $A d S_{n}$ directions and $\tau_{ \pm}$and $\sigma_{ \pm}$depend only on the coordinates of $M^{11-n}$. Moreover, the remaining independent KSEs on $\tau_{ \pm}$and $\sigma_{ \pm}$consist of those that one can find by the naive restriction of the KSEs of $\phi_{ \pm}$on $M^{11-n}$, together with the introduction of the above mentioned new algebraic KSEs one for each $\tau_{ \pm}$and $\sigma_{ \pm}$. Therefore, the independent KSEs can be arranged in four different sets, and each set contains two equations.

The derivation of the formula (1.1) for the $A d S_{n}$ backgrounds, $n>2$, is based on the observation that there are interwining Clifford algebra operators which allow for a given solution to one of the four sets of KSEs to produce solutions to the other three sets. 
After taking into account all such Clifford algebra operations, the formula (1.1) can be established.

The proof of (1.2) for $A d S_{n}, n>2$, requires the choice of appropriate Dirac-like operators $\mathscr{D}^{( \pm)}$on $M^{11-n}$. These are linear combinations of the Dirac operators derived from the gravitino KSEs, and the new algebraic KSEs which arise on $M^{11-n}$ after integrating along the $A d S_{n}$ directions. Then assuming that $\mathscr{D}^{(+)} \chi_{ \pm}=0$, where $\chi_{+}$is either $\sigma_{+}$or $\tau_{+}$, one schematically establishes

$$
D^{2}\left\|\chi_{+}\right\|^{2}+n A^{-1} \partial^{i} A \partial_{i}\left\|\chi_{+}\right\|^{2}=2\left\langle\mathbb{D}^{(+)} \chi, \mathbb{D}^{(+)} \chi_{+}\right\rangle+2 \frac{9 n-18}{11-n}\left\|\mathcal{C}^{(+)} \chi_{+}\right\|^{2},
$$

where $\mathbb{D}^{(+)}$is the gravitino KSE appropriately modified by the new algebraic KSE and $\mathcal{C}^{(+)}$ is the new algebraic KSE. A similar formula holds for the $\sigma_{-}$and $\tau_{-}$spinors. Provided that the requirements for the maximum principle to hold are satisfied by the fields and $M^{11-n}$, i.e. for smooth fields and $M^{11-n}$ compact without boundary, one concludes that the only solutions to the above equation is that $\left\|\chi_{+}\right\|$is constant and $\chi_{+}$satisfies the KSEs. This demonstrates a new type of Lichnerowicz theorems for $\mathscr{D}^{( \pm)}$. These theorems establish an 1-1 correspondence between the zero modes of $\mathscr{D}^{( \pm)}$and Killing spinors. Using these, (1.2) follows from (1.1) and a counting of the multiplicity of zero modes of $\mathscr{D}^{( \pm)}$. This is done by identifying the Clifford algebra operators which commute with $\mathscr{D}^{( \pm)}$.

For the proof of the number of supersymmetries preserved by flat backgrounds $\mathbb{R}^{n-1,1}$ in (1.3), it suffices to investigate the KSEs that arise for the $A d S_{n}$ backgrounds in the limit of infinite AdS radius. All our local computations are smooth in this limit and so these computations carry through to all flat $n>2$ cases. Using this, one can establish the independent KSEs on $M^{11-n}$ for flat backgrounds from those of the $A d S_{n}$ solutions. However there is a difference in that the KSEs for $\sigma_{ \pm}$and $\tau_{ \pm}$spinors become identical at the limit of infinite AdS radius. As a result the $\sigma_{ \pm}$and $\tau_{ \pm}$are not linearly independent and so the counting of supersymmetries differs from that of $A d S_{n}$ backgrounds. After taking into account the Clifford algebra operators which intertwine between and those that commute with the KSEs, one can establish (1.3). Furthermore for flat backgrounds, some of the regularity assumptions used to establish the Lichnerowicz type theorems for the $A d S_{n}$ backgrounds do not hold. As a result, there is no analogue of the formula (1.2) for flat backgrounds. For the same reason, one cannot establish that the number of supersymmetries preserved by $\mathbb{R}^{1,1}$ backgrounds is even as this, for $A d S_{2}$ backgrounds, requires the use of global arguments.

This paper has been organized as follows. In section 2, we describe all $A d S_{n}$ and $\mathbb{R}^{n-1,1}$ backgrounds as near horizon geometries and summarize some of the results of [22] on 11-dimensional horizons which are essential for the investigation that follows. In section 3, we solve the KSEs for the $A d S_{2}$ backgrounds along the $A d S_{2}$ directions. In section 4 we demonstrate two new Lichnerowicz type theorems for $A d S_{2}$ backgrounds utilizing the maximum principle and verify that such backgrounds preserve an even number of supersymmetries. In sections 5, 7 and 9, we solve the KSEs for $A d S_{3}, A d S_{4}$ and $A d S_{5}$ backgrounds verifying (1.1), respectively. In sections 6,8 and 10, we prove new Lichnerowicz type theorems for $A d S_{3}, A d S_{4}$ and $A d S_{5}$ backgrounds verifying (1.2), respectively. In 
section 11, we investigate the factorization of the Killing spinors of AdS backgrounds. In section 12 , we prove (1.2) for $\mathbb{R}^{n-1,1}$ backgrounds. We state our conclusions and describe some preliminary results on the geometry of $A d S_{n}$ and $\mathbb{R}^{n-1,1}$ backgrounds in section 13.

\section{AdS backgrounds and near horizon geometries}

\subsection{Warped AdS and flat backgrounds}

To systematize the investigation of warped AdS and Minkowski backgrounds in 11-dimensional supergravity, $A d S_{n} \times{ }_{w} M^{11-n}$ and $\mathbb{R}^{n-1,1} \times_{w} M^{11-n}$, respectively, it is convenient to express them as near horizon geometries. This has already been suggested in [20], where a preliminary analysis has been carried out. In particular, the most general form of the metric and 4-form flux which includes all these backgrounds can be written in Gaussian null coordinates $[24,25]$ as

$$
\begin{aligned}
d s^{2} & =2 \mathbf{e}^{+} \mathbf{e}^{-}+\delta_{i j} \mathbf{e}^{i} \mathbf{e}^{j}=2 d u\left(d r+r h-\frac{1}{2} r^{2} \Delta d u\right)+d s^{2}(\mathcal{S}), \\
F & =\mathbf{e}^{+} \wedge \mathbf{e}^{-} \wedge Y+r \mathbf{e}^{+} \wedge d_{h} Y+X
\end{aligned}
$$

where we have introduced the frame

$$
\mathbf{e}^{+}=d u, \quad \mathbf{e}^{-}=d r+r h-\frac{1}{2} r^{2} \Delta d u, \quad \mathbf{e}^{i}=e_{J}^{i} d y^{J} ; \quad g_{I J}=\delta_{i j} e_{I}^{i} e_{J}^{j},
$$

and

$$
d s^{2}(\mathcal{S})=\delta_{i j} \mathbf{e}^{i} \mathbf{e}^{j},
$$

is the metric on the space $\mathcal{S}$ transverse to the lightcone directions given by $r=u=0$. The dependence on the coordinates $r, u$ is given explicitly and $d_{h} Y=d Y-h \wedge Y$. In addition, $\Delta, h, Y$ and $X$ are a 0 -form, 1-form, 2-form and a 4 -form on $\mathcal{S}$ and depend only on the coordinates $y$ of $\mathcal{S}$. We choose the frame indices $i=1,2,3,4,6,7,8,9, \sharp$ and we follow the conventions of [23]. The form of the fields in (2.1) is the same as that of near horizon geometries investigated in [21-23].

There are many advantages to describe the AdS backgrounds in terms of near horizon geometries as in (2.1). One of them is that some of the results obtained for near horizon geometries in [21-23] can be directly applied here, eg one can use the integrability of the light-cone directions for horizons to reduce the problem to the identification of the geometry of near horizon sections $\mathcal{S}$. This is a problem in Riemannian geometry which is easier to solve. To describe each $A d S_{n} \times_{w} M$ case separately, for $n=2, \ldots, 11$, some additional restrictions have to be imposed on the near horizon fields (2.1). This is because the $A d S_{n} \times_{w} M^{11-n}$ backgrounds are invariant under the $\mathrm{SO}(n-1,2)$ isometry group of $A d S_{n}$. Imposing this symmetry, we have the following. 


\subsection{1 $\quad \operatorname{AdS}_{2} \times_{w} M^{9}$}

The metric and fluxes are

$$
\begin{aligned}
d s^{2} & =2 d u\left(d r+r h-\frac{1}{2} r^{2} \Delta d u\right)+d s^{2}\left(M^{9}\right), \\
F & =\mathbf{e}^{+} \wedge \mathbf{e}^{-} \wedge Y+X
\end{aligned}
$$

with

$$
h=-2 A^{-1} d A=\Delta^{-1} d \Delta, \quad d_{h} Y=0,
$$

where $A, Y$ and $X$ are a 0 -form, 2-form and a 4 -form on $M^{9}=\mathcal{S}$, respectively. Observe that $d h=0$.

\subsection{2 $\quad A d S_{3} \times_{w} M^{8}$}

The fields are

$$
\begin{aligned}
d s^{2} & =2 d u(d r+r h)+A^{2} d z^{2}+d s^{2}\left(M^{8}\right), \\
F & =\mathbf{e}^{+} \wedge \mathbf{e}^{-} \wedge d z \wedge Q+X,
\end{aligned}
$$

with

$$
h=-\frac{2}{\ell} d z-2 A^{-1} d A, \quad \Delta=0, \quad Y=d z \wedge Q, \quad d_{h} Y=0
$$

where $A, Q, X$ are a 0 -form, 1-form and a 4 -form of $M^{8}$, respectively, and depend only on the coordinates of $M^{8} . \ell$ is the radius of AdS.

\subsection{3 $\quad \operatorname{AdS}_{4} \times_{w} M^{7}$}

The fields are

$$
\begin{aligned}
d s^{2} & =2 d u(d r+r h)+A^{2}\left(d z^{2}+e^{2 z / \ell} d x^{2}\right)+d s^{2}\left(M^{7}\right) \\
F & =e^{z / \ell} A^{2} S \mathbf{e}^{+} \wedge \mathbf{e}^{-} \wedge d z \wedge d x+X
\end{aligned}
$$

with

$$
h=-\frac{2}{\ell} d z-2 A^{-1} d A, \quad \Delta=0, \quad Y=e^{z / \ell} A^{2} S d z \wedge d x, \quad d_{h} Y=0
$$

where $A, S, X$ are a 0 -form, 0 -form and a 4 -form of $M^{7}$, respectively, and depend only on the coordinates of $M^{7} . \ell$ is the radius of AdS.

\subsection{4 $\quad \operatorname{AdS}_{n} \times_{w} M^{11-n}, n>4$}

The fields of the rest of the backgrounds are

$$
\begin{aligned}
d s^{2} & =2 d u(d r+r h)+A^{2}\left(d z^{2}+e^{2 z / \ell} \sum_{a=1}^{n-3}\left(d x^{a}\right)^{2}\right)+d s^{2}\left(M^{11-n}\right), \\
F & =X
\end{aligned}
$$


with

$$
h=-\frac{2}{\ell} d z-2 A^{-1} d A, \quad \Delta=0, \quad Y=0,
$$

where $A, X$ are a 0 -form and a 4 -form of $M^{11-n}$, respectively, and depend only on the coordinates of $M^{11-n}$. $\ell$ is the radius of AdS.

Observe that in all the above backgrounds $A d S_{n} \times_{w} M^{11-n}$, we have that $\mathcal{S}=H^{n-2} \times_{w}$ $M^{11-n}$, i.e. $\mathcal{S}$ is the warped product of hyperbolic (n-2)-dimensional space $H^{n-2}$ with the transverse space $M^{11-n}$ of $A d S_{n}$.

\subsection{5 $\mathbb{R}^{n-1,1} \times_{w} M^{11-n}$}

Another advantage of the universal ansatz (2.1) is that it includes the warped $\mathbb{R}^{n-1,1} \times_{w}$ $M^{11-n}$ backgrounds. These arise in the limit of large AdS radius, $\ell \rightarrow \infty$. This limit is smooth in all our calculations and so our AdS results can be adapted to $\mathbb{R}^{n-1,1} \times{ }_{w} M^{11-n}$ backgrounds. However, many properties of the $A d S_{n} \times{ }_{w} M^{11-n}$ backgrounds do not hold in the limit of $\mathbb{R}^{n-1,1} \times_{w} M^{11-n}$ backgrounds and some care must be taken when taking this limit.

\subsection{Bianchi identities and field equations}

The fields of $A d S_{n} \times{ }_{w} M^{11-n}$ backgrounds are restricted by the field equations and Bianchi identities of 11-dimensional supergravity [26]. Before we proceed to apply these to each $A d S_{n} \times_{w} M$ background separately, it is convenient to decompose them for the universal ansatz (2.1) along the light-cone directions and the rest. In preparation for the applications to $A d S_{n} \times{ }_{w} M^{11-n}$, we shall also impose that $d_{h} Y=0$ and $d h=0$ which are satisfied for all these backgrounds. The end result of the decomposition of the field equations and later the KSEs along the light-cone and $\mathcal{S}$ directions is to reduce the problem on $\mathcal{S}$ as the light-cone directions are integrable. In particular, we find the following.

The decomposition of the Bianchi identity of $F, d F=0$, for the universal ansatz (2.1) yields

$$
d X=0
$$

i.e. $X$ is a closed form on $\mathcal{S}$.

Similarly, the field equation

$$
d \star_{11} F-\frac{1}{2} F \wedge F=0,
$$

of the 3-form gauge potential yields

$$
\tilde{\nabla}^{i} X_{i \ell_{1} \ell_{2} \ell_{3}}+=h^{i} X_{i \ell_{1} \ell_{2} \ell_{3}}-\frac{1}{48} \epsilon_{\ell_{1} \ell_{2} \ell_{3}}{ }^{q_{1} q_{2} q_{3} q_{4} q_{5} q_{6}} Y_{q_{1} q_{2}} X_{q_{3} q_{4} q_{5} q_{6}}
$$

and

$$
\tilde{\nabla}^{j} Y_{j i}-\frac{1}{1152} \epsilon_{i}^{q_{1} q_{2} q_{3} q_{4} q_{5} q_{6} q_{7} q_{8}} X_{q_{1} q_{2} q_{3} q_{4}} X_{q_{5} q_{6} q_{7} q_{8}}=0,
$$

where $\tilde{\nabla}$ is the Levi-Civita connection of the metric $d s^{2}(\mathcal{S})$ on $\mathcal{S}$. 
The Einstein equation

$$
R_{M N}=\frac{1}{12} F_{M N}^{2}-\frac{1}{144} g_{M N} F^{2} .
$$

of 11-dimensional supergravity decomposes into a number of components. In particular along $\mathcal{S}$, one finds

$$
\tilde{R}_{i j}+\tilde{\nabla}_{(i} h_{j)}-\frac{1}{2} h_{i} h_{j}=-\frac{1}{2} Y_{i j}^{2}+\frac{1}{12} X_{i j}^{2}+\delta_{i j}\left(\frac{1}{12} Y^{2}-\frac{1}{144} X^{2}\right),
$$

where $\tilde{R}_{i j}$ is the Ricci tensor of $\mathcal{S}$. The +- component of the Einstein equation gives

$$
\tilde{\nabla}^{i} h_{i}=2 \Delta+h^{2}-\frac{1}{3} Y^{2}-\frac{1}{72} X^{2}
$$

Similarly, the ++ and $+i$ components of the Einstein equation can be expressed as

$$
\frac{1}{2} \tilde{\nabla}^{i} \tilde{\nabla}_{i} \Delta-\frac{3}{2} h^{i} \tilde{\nabla}_{i} \Delta-\frac{1}{2} \Delta \tilde{\nabla}^{i} h_{i}+\Delta h^{2}=0,
$$

and

$$
-\tilde{\nabla}_{i} \Delta+\Delta h_{i}=0
$$

respectively. The last equation implies that if $\Delta \neq 0$, which is the case for only the $A d S_{2} \times_{w} M^{9}$ backgrounds, then $h=\Delta^{-1} d \Delta$.

As has been explained in [22], the ++ and the $+i$ components of the Einstein equations, (2.19) and (2.20), respectively, are not independent but they hold as a consequence of (2.12), the 3 -form field equations (2.14) and (2.15) and the components of the Einstein equation in (2.17) and (2.18). This does not make use of supersymmetry, or any other assumptions on $\mathcal{S}$. Hence, the conditions on $d s^{2}(\mathcal{S}), \Delta, h, Y$ and $X$ simplify to $(2.12),(2.14),(2.15),(2.17)$ and (2.18).

\subsection{Killing spinor equations}

Another advantage of using the universal ansatz (2.1) is that one can apply the results obtained for near horizon geometries in $[22,23]$ to integrate the KSEs

$$
\nabla_{M} \epsilon+\left(-\frac{1}{288} \not F_{M}+\frac{1}{36} \not F_{M}\right) \epsilon=0
$$

of 11-dimensional supergravity [26], where $\nabla$ is the spacetime Levi-Civita connection. In particular, it is known that the KSEs evaluated on the universal ansatz (2.1) are integrable along the light-cone directions. The analysis that follows is carried out in generality. In particular, we do not put any restrictions on the form of either the Killing spinor, or of the fields. Moreover, we do not impose any other additional restrictions, like for example the bi-linear matching condition. Furthermore, the calculation is local and applies to all $A d S_{n} \times{ }_{w} M$ backgrounds. 


\subsubsection{Light-cone integrability and independent KSEs}

After integrating along the light-cone directions following [21, 22], one can write the Killing spinor $\epsilon$ as

$$
\epsilon=\epsilon_{+}+\epsilon_{-}, \quad \Gamma_{ \pm} \epsilon_{ \pm}=0
$$

with

$$
\epsilon_{+}=\phi_{+}+u \Gamma_{+} \Theta_{-} \phi_{-}, \quad \epsilon_{-}=\phi_{-}+r \Gamma_{-} \Theta_{+} \epsilon_{+},
$$

where

$$
\Theta_{ \pm}=\left(\frac{1}{4} \not h+\frac{1}{288} X \pm \frac{1}{12} Y\right)
$$

$\Gamma_{ \pm}$are light-cone projections, and $\phi_{ \pm}=\phi_{ \pm}(y)$ do not depend on $r$ or $u$ and depend only on the coordinates of $\mathcal{S}$. For future reference, we write

$$
\epsilon\left(\phi_{-}, \phi_{+}\right)=\phi_{+}+u \Gamma_{+} \Theta_{-} \phi_{-}+\phi_{-}+r \Gamma_{-} \Theta_{+}\left(\phi_{+}+u \Gamma_{+} \Theta_{-} \phi_{-}\right) .
$$

Furthermore, one can show that the only remaining independent KSEs are

$$
\nabla_{i}^{( \pm)} \phi_{ \pm} \equiv \tilde{\nabla}_{i} \phi_{ \pm}+\Psi_{i}^{( \pm)} \phi_{ \pm}=0
$$

where

$$
\Psi_{i}^{( \pm)}=\mp \frac{1}{4} h_{i}-\frac{1}{288} \not X_{i}+\frac{1}{36} X_{i} \pm \frac{1}{24} \not Y_{i} \mp \frac{1}{6} Y_{i},
$$

and that if $\phi_{-}$is a solution of the KSEs, $\nabla_{i}^{(-)} \phi_{-}=0$, then

$$
\phi_{+} \equiv \Gamma_{+} \Theta_{-} \phi_{-}
$$

is also a solution, $\nabla_{i}^{(+)} \phi_{+}=0$. These results follow after substituting the spinor (2.22) into the KSEs (2.21) and after an extensive use of the Bianchi identities and field equations.

The substitution of (2.22) into the KSEs (2.21) yields a large number of integrability conditions that are satisfied after an extensive use of the Bianchi identities, field equations and the independent KSEs (2.26). However in what follows, it is instructive to include two of these integrability conditions. Although these are not independent, nevertheless they are useful in the solution of the KSEs along the remaining AdS directions. After imposing $d h=d_{h} Y=0$, which are satisfied for all $A d S_{n} \times{ }_{w} M^{11-n}$ backgrounds, we have

$$
\left(\frac{1}{2} \Delta+2\left(\frac{1}{4} \not h-\frac{1}{288} \not X+\frac{1}{12} Y\right) \Theta_{+}\right) \phi_{+}=0,
$$

and

$$
\left(-\frac{1}{2} \Delta+2\left(-\frac{1}{4} \not h+\frac{1}{288} \not X+\frac{1}{12} Y\right) \Theta_{-}\right) \phi_{-}=0 .
$$

To summarize, the independent KSEs that have to be solved to find the supersymmetric $A d S_{n} \times{ }_{w} M^{11-n}$ backgrounds are (2.26). In what follows, we shall demonstrate that these can be integrated along the remaining AdS directions in $A d S_{n} \times_{w} M^{11-n}$. 


\section{$3 \quad A d S_{2}$ : local analysis}

\subsection{Field equations}

In this case $M^{9}=\mathcal{S}$, the fields on $\mathcal{S}$ are

$$
d s^{2}(\mathcal{S})=d s^{2}\left(M^{9}\right), \quad \tilde{F}^{2}=Y, \quad \tilde{F}^{4}=X, \quad h=-2 A^{-1} d A .
$$

Next, it follows from (2.20) that $h=\Delta^{-1} d \Delta$ and so

$$
\Delta=\ell^{-2} A^{-2},
$$

where $\ell$ is the radius of $A d S_{2}$. Furthermore, the Bianchi identity for $F$ and $d_{h} Y=0$ imply that

$$
d X=0, \quad d\left(A^{2} Y\right)=0 .
$$

The remaining independent field equations are

$$
\begin{aligned}
\tilde{\nabla}^{i}\left(A^{2} X_{i \ell_{1} \ell_{2} \ell_{3}}\right) & =-\frac{A^{2}}{48} \epsilon_{\ell_{1} \ell_{2} \ell_{3}}{ }^{{ }_{1} q_{2} q_{3} q_{4} q_{5} q_{6}} Y_{q_{1} q_{2}} X_{q_{3} q_{4} q_{5} q_{6}}, \\
\tilde{R}_{i j}-\tilde{\nabla}_{i} \partial_{j} \log A^{2}-\frac{1}{2} \partial_{i} \log A^{2} \partial_{j} \log A^{2} & =-\frac{1}{2} Y_{i j}^{2}+\frac{1}{12} X_{i j}^{2}+\delta_{i j}\left(\frac{1}{12} Y^{2}-\frac{1}{144} X^{2}\right), \\
\tilde{\nabla}^{i} \partial_{i} \log A & =-\frac{A^{-2}}{\ell^{2}}-2 \partial^{i} \log A \partial_{i} \log A+\frac{1}{6} Y^{2}+\frac{1}{144} X^{2},
\end{aligned}
$$

and (2.15) which remains unmodified.

\subsubsection{The warp factor $A$ is nowhere vanishing}

Suppose that $M^{9}$ is path connected, the fields are smooth and $A$ is not identical to zero but it may vanish at some points in $M^{9}$. First let $p \in M^{9}$ such that $A(p)=0$ and a point $q \in M^{9}$ such that $A(q) \neq 0$. Without loss of generality we take $A(q)>0$, otherwise one can consider $-A$ for the argument that follows. Moreover consider a smooth path $\gamma$ with $\gamma(0)=q$ and $\gamma(1)=p$, and the function $f=A \circ \gamma$. As $A$ is continuous, there is a neighborhood $U$ of $q$ such that $\left.A\right|_{U} \neq 0$. Next consider, the set

$$
V=\{a \in[0, \infty): \quad f(t) \neq 0, \quad \forall t \in[0, a]\} .
$$

$V$ is not empty as $0 \in V$, and $W \subset V$ where $W=\{t \in[0,1]: \gamma(t) \in U\}$. As $V$ is bounded by 1 , it has a supremum $b$. Continuity requires that $f(b)=0$ and there is a sequence $\left\{t_{n}\right\}$ in $V$ which converges at $b$.

Next restricting (3.6) at $\gamma$ and multiplying it with $f^{2}\left(t_{n}\right) \neq 0$ for some finite $n$, we find

$$
f\left(t_{n}\right) \nabla^{2} A+\partial_{i} A \partial^{i} A+\frac{1}{\ell^{2}}+\frac{f\left(t_{n}\right)^{2}}{6} Y^{2}+\frac{f\left(t_{n}\right)^{2}}{144} X^{2}=0 .
$$

Now as $n \rightarrow \infty, f\left(t_{n}\right)$ approaches zero and becomes very small while the derivatives of $A$ and the fluxes are smooth and so their values are bounded when restricted on $[0,1]$ which is compact. As a result, (3.6) cannot be satisfied very close to $\gamma(b)$ as the term $\frac{1}{\ell^{2}}$ which depends on the radius of AdS cannot be arbitrary close to zero for any $\ell^{2}<\infty$. Thus $A$ cannot vanish. Later, we shall see that $A^{2}$ is related to the length of a parallel spinor in all $A d S_{k}$ cases which again confirms that $A$ is nowhere vanishing. 


\subsection{Killing spinor equations}

The KSEs on $\mathcal{S}=M^{9}$ are

$$
\nabla_{i}^{( \pm)} \phi_{ \pm} \equiv \tilde{\nabla}_{i} \phi_{ \pm}+\Psi_{i}^{( \pm)} \phi_{ \pm}=0
$$

where

$$
\Psi_{i}^{( \pm)}= \pm \frac{1}{4} \partial_{i} \log A^{2}-\frac{1}{288} \not X_{i}+\frac{1}{36} \not X_{i} \pm \frac{1}{24} \not Y_{i} \mp \frac{1}{6} Y_{i}
$$

Furthermore from the general results on horizons, if $\phi_{-}$is a solution of the KSEs, $\nabla_{i}^{(-)} \phi_{-}=$ 0 , then

$$
\phi_{+} \equiv \Gamma_{+} \Theta_{-} \phi_{-}
$$

is also a solution, $\nabla_{i}^{(+)} \phi_{+}=0$, where

$$
\Theta_{ \pm}=\left(-\frac{1}{4} \not \partial \log A^{2}+\frac{1}{288} \not X \pm \frac{1}{12} Y\right) .
$$

It is not apparent that $\phi_{+}=\Gamma_{+} \Theta_{-} \phi_{-} \neq 0$. In particular, $\phi_{-}$can be in the kernel of $\Theta_{-}$. In the $A d S_{2}$ case, it has been shown in [23] that if $M^{9}$ is compact without boundary, then $\operatorname{Ker} \Theta_{-}=\{0\}$ and so $\phi_{+} \neq 0$ which leads to the enhancement of supersymmetry.

\section{$4 \quad A d S_{2}$ : global analysis}

To prove that the number of supersymmetries preserved by $A d S_{2}$ backgrounds is even, it is required to establish Lichnerowicz type theorems relating the Killing spinors $\phi_{ \pm}$, $\nabla^{( \pm)} \phi_{ \pm}=0$, to the zero modes of the Dirac-like operators

$$
\mathcal{D}^{( \pm)}=\Gamma^{i} \tilde{\nabla}_{i}+\Psi^{( \pm)}
$$

where

$$
\Psi^{( \pm)}=\Gamma^{i} \Psi_{i}^{( \pm)}= \pm \frac{1}{4} \not \partial \log A^{2}+\frac{1}{96} \not X \pm \frac{1}{8} Y
$$

Such theorems have been demonstrated in the context of near horizon geometries in [23]. Since the $A d S_{2}$ backgrounds are a special case of near horizon geometries, the result follows. However, there is a difference. For $A d S_{2}$ backgrounds both Lichnerowicz type theorems can be shown using the maximum principle which has an advantage relative to a partial integration formula used in [23]. This is because one also finds a restriction on the length of the spinors.

\subsection{A Lichnerowicz type of theorem for $\mathcal{D}^{(+)}$}

The proof of this theorem is identical to that in [23] and so we shall not give details. It is clear that if $\tilde{\nabla}^{(+)} \phi_{+}=0$, then $\phi_{+}$is a zero mode of the $\mathcal{D}^{(+)}$operator. To prove the 
converse, let us assume that $\mathcal{D}^{(+)} \phi_{+}=0$. Then after using the field equations and Bianchi identities, one obtains

$$
\tilde{\nabla}^{i} \tilde{\nabla}_{i}\left\|\phi_{+}\right\|^{2}+\partial^{i} \log A^{2} \tilde{\nabla}_{i}\left\|\phi_{+}\right\|^{2}=2\left\langle\tilde{\nabla}^{(+) i} \phi_{+}, \tilde{\nabla}_{i}^{(+)} \phi_{+}\right\rangle
$$

Assuming that the requirements for applying of the maximum principle hold ${ }^{2}$ on the function $\left\|\phi_{+}\right\|^{2}$, eg $M^{9}$ is compact and the fields are smooth, one finds that the only solutions to the above equation are

$$
\mathcal{D}^{(+)} \phi_{+}=0 \Longleftrightarrow \tilde{\nabla}_{i}^{(+)} \phi_{+}=0
$$

and

$$
\left\|\phi_{+}\right\|=\text {const }
$$

This establishes the theorem.

\subsection{A Lichnerowicz theorem for $\mathcal{D}^{(-)}$}

The proof of the Lichnerowicz type theorem for the $\mathcal{D}^{(-)}$operator is done in a similar fashion as that for $\mathcal{D}^{(+)}$. It is clear that if $\tilde{\nabla}^{(-)} \phi_{-}=0$, then $\phi_{-}$is a zero mode of the $\mathcal{D}^{(-)}$operator. To prove the converse, let us assume that $\mathcal{D}^{(-)} \phi_{-}=0$. Then after using the field equations and Bianchi identities, one obtains

$$
\tilde{\nabla}^{i} \tilde{\nabla}_{i}\left\|\phi_{-}\right\|^{2}+h^{i} \tilde{\nabla}_{i}\left\|\phi_{-}\right\|^{2}+\left(\tilde{\nabla}^{i} h_{i}\right)\left\|\phi_{-}\right\|^{2}=2\left\langle\tilde{\nabla}^{(-) i} \phi_{-}, \tilde{\nabla}_{i}^{(-)} \phi_{-}\right\rangle .
$$

For the $A d S_{2}$ solutions, $h=\Delta^{-1} d \Delta$, and this expression can be rewritten as

$$
\tilde{\nabla}^{i} \tilde{\nabla}_{i}\left(\Delta\left\|\phi_{-}\right\|^{2}\right)-h^{i} \tilde{\nabla}_{i}\left(\Delta\left\|\phi_{-}\right\|^{2}\right)=2 \Delta\left\langle\tilde{\nabla}^{(-) i} \phi_{-}, \tilde{\nabla}_{i}^{(-)} \phi_{-}\right\rangle
$$

Since $\Delta$ is nowhere zero applying the maximum principle on the function $\Delta\left\|\phi_{-}\right\|^{2}$, one concludes that

$$
\mathcal{D}^{(-)} \phi_{-}=0 \Longleftrightarrow \tilde{\nabla}_{i}^{(-)} \phi_{-}=0
$$

and

$$
\Delta\left\|\phi_{-}\right\|^{2}=\text { const } .
$$

This establishes the 1-1 correspondence between Killing spinors and the zero modes of the Dirac-like operator $\mathcal{D}^{(-)}$.

\footnotetext{
${ }^{2}$ From now on whenever we apply the maximum principle we shall assume that all the requirements on the fields and the associated manifold hold.
} 


\subsection{Counting supersymmetries}

One of the applications of the global analysis above is to prove that $A d S_{2} \times{ }_{w} M$ backgrounds preserve $2 k, 0<k<15$, supersymmetries. The proof that $A d S_{2} \times_{w} M^{9}$ backgrounds preserve an even number of supersymmetries is similar to that for near horizon geometries established in [23]. The main point of the proof is that the $\mathcal{D}^{(-)}$operator has the same principal symbol as the standard Dirac operator on the 9-dimensional manifold $M^{9}$. As a result they have the same index which in this case vanishes, as the index of the Dirac operator on compact without boundary odd dimensional manifolds is zero. Moreover, one can show that the dimension of the Kernel of $\mathcal{D}^{(+)}$operator is the same as that of $\left(\mathcal{D}^{(-)}\right)^{\dagger}$, the adjoint of $\mathcal{D}^{(-)}$. Now the number of supersymmetries $N$ preserved by the $A d S_{2} \times_{w} M$ backgrounds is

$$
\begin{aligned}
N & =\operatorname{dim} \operatorname{Ker} \mathcal{D}^{(-)}+\operatorname{dim} \operatorname{Ker} \mathcal{D}^{(+)}=\operatorname{dim} \operatorname{Ker} \mathcal{D}^{(-)}+\operatorname{dim} \operatorname{Ker}\left(\mathcal{D}^{(-)}\right)^{\dagger} \\
& =2 \operatorname{dim} \operatorname{Ker} \mathcal{D}^{(-)}=2 k,
\end{aligned}
$$

where we have used that $\operatorname{dim} \operatorname{Ker} \mathcal{D}^{(-)}=\operatorname{dim} \operatorname{Ker}\left(\mathcal{D}^{(-)}\right)^{\dagger}$ because the index of $\mathcal{D}^{(-)}$is zero [27].

The restriction $0<k<15$ in the range of $k$ arises because of the results of [18] and [16] which rule out the existence of $A d S_{2} \times{ }_{w} M$ backgrounds preserving 30 and 32 supersymmetries, respectively.

\section{$5 \quad \mathrm{AdS}_{3}$ : local Analysis}

\subsection{Bianchi identities and field equations}

The metric on $\mathcal{S}=\mathbb{R} \times{ }_{w} M^{8}$ and the form field strengths are

$$
\begin{aligned}
d s^{2}(\mathcal{S}) & =A^{2} d z^{2}+d s^{2}\left(M^{8}\right), & d s^{2}\left(M^{8}\right) & =\delta_{i j} \mathbf{e}^{i} \mathbf{e}^{j}, \\
Y & =A^{-1} \mathbf{e}^{z} \wedge Q, & \tilde{F} & =X .
\end{aligned}
$$

Next the Bianchi identity for $F$ implies that

$$
d\left(A^{2} Q\right)=0, \quad d X=0 .
$$

Let $D$ be the Levi-Civita connection ${ }^{3}$ on $M^{8}$ and $d \operatorname{vol}(\mathcal{S})=\mathbf{e}^{z} \wedge d \operatorname{vol}\left(M^{8}\right)$. Then, we find that the field equations (2.14) and (2.15) of the 4 -form $F$ reduce on $M^{8}$ as

$$
D^{k} X_{k i_{1} i_{2} i_{3}}=-3 A^{-1} D^{k} A X_{k i_{1} i_{2} i_{3}}+\frac{1}{24} A^{-1} \epsilon_{i_{1} i_{2} i_{3}}{ }^{j_{1} j_{2} j_{3} j_{4} j_{5}} Q_{j_{1}} X_{j_{2} j_{3} j_{4} j_{5}}
$$

and

$$
D^{k}\left(A^{-1} Q_{k}\right)+\frac{1}{1152} \epsilon^{i_{1} i_{2} i_{3} i_{4} i_{5} i_{6} i_{7} i_{8}} X_{i_{1} i_{2} i_{3} i_{4}} X_{i_{5} i_{6} i_{7} i_{8}}=0 .
$$

\footnotetext{
${ }^{3}$ From here on $D$ will denote the Levi-Civita connection on $M^{11-n}$ and the latin indices $i, j, k, \ell$ are frame indices for $M^{11-n}$.
} 
We also reduce the Einstein equation (2.17). From the $z z$ component one obtains

$$
A^{-1} D^{k} D_{k} A+2 A^{-2} D^{k} A D_{k} A+\frac{2}{\ell^{2}} A^{-2}=\frac{1}{3} A^{-2} Q^{2}+\frac{1}{144} X^{2},
$$

and from the $i, j$ component, one has

$$
R_{i j}^{(8)}=3 A^{-1} D_{i} D_{j} A-\frac{1}{2} A^{-2} Q_{i} Q_{j}+\frac{1}{12} X_{i j}^{2}+\delta_{i j}\left(\frac{1}{6} A^{-2} Q^{2}-\frac{1}{144} X^{2}\right),
$$

where $R^{(8)}$ denotes the Ricci tensor of $M^{8}$.

\subsubsection{The warp factor $A$ is nowhere vanishing}

Before proceeding with the analysis of the supersymmetry, we shall first examine whether or not $A$ can vanish somewhere on $M^{8}$. For this, we use a similar set up as for the proof of $A \neq 0$ for the $A d S_{2}$ case. In particular after assuming that there are points $p$ and $q$ with $A(p)=0$ and $A(q) \neq 0$, constructing a path $\gamma$ between $p=\gamma(1)$ and $q=\gamma(0)$, and arguing the existence of a sequence $t_{n}$ such that $\lim _{n \rightarrow \infty} f\left(t_{n}\right)=0$ where $f\left(t_{n}\right)=A\left(\gamma\left(t_{n}\right)\right) \neq 0$, we have from (5.6) and (5.5) that

$$
f^{2}\left(t_{n}\right) R^{(8)}=-6 D^{k} A D_{k} A-\frac{6}{\ell^{2}}+\frac{11}{6} Q^{2}+\frac{7}{144} f\left(t_{n}\right)^{2} X^{2},
$$

and

$$
f\left(t_{n}\right) D^{k} D_{k} A+2 D^{k} A D_{k} A+\frac{2}{\ell^{2}}=\frac{1}{3} Q^{2}+\frac{1}{144} f\left(t_{n}\right)^{2} X^{2} .
$$

Eliminating the $Q^{2}$ term from the second equation using the first, we find

$$
11 f\left(t_{n}\right) D^{i} D_{i} A+16 D^{i} A D_{i} A+\frac{16}{\ell^{2}}=f^{2}\left(t_{n}\right) R^{(8)}+\frac{1}{36} f^{2}\left(t_{n}\right) X^{2} .
$$

Assuming regularity for all the data and that $M^{8}$ is path connected, an argument similar to that of the $A d S_{2}$ case implies that the above equation cannot be satisfied if $A$ vanishes. Therefore $A \neq 0$ everywhere on $M^{8}$.

\subsection{Killing spinor equations}

To reduce the KSEs from $\mathcal{S}$ to $M^{8}$, we first decompose (2.26) along the $z$-direction to find

$$
\partial_{z} \phi_{ \pm}=\Xi^{( \pm)} \phi_{ \pm}
$$

where

$$
\Xi^{( \pm)}=-\frac{1}{2} \Gamma_{z} \not \partial \mp \frac{1}{2 \ell}+\frac{A}{288} \Gamma_{z} \not \pm \frac{1}{6} \not,
$$

$\partial_{z} \equiv \frac{\partial}{\partial z}$ and $\Gamma_{z}$ denotes the frame gamma matrix from here on. The KSEs (2.26) along the remaining directions can be written as

$$
D_{i}^{( \pm)} \phi_{ \pm}=0
$$


where

$$
D_{i}^{( \pm)}=D_{i} \pm \frac{1}{2} A^{-1} D_{i} A-\frac{1}{288} \not X_{i}+\frac{1}{36} X_{i} \mp \frac{1}{12} A^{-1} \Gamma_{z} \not Q_{i} \pm \frac{1}{6} A^{-1} \Gamma_{z} Q_{i} .
$$

Before proceeding further, it is useful to evaluate the following two integrability conditions

$$
\Gamma^{j}\left(D_{j} D_{i}-D_{i} D_{j}\right) \phi_{ \pm}=\frac{1}{2} R^{(8)}{ }_{i j} \Gamma^{j} \phi_{ \pm}
$$

and

$$
\left(\partial_{z} D_{i}-D_{i} \partial_{z}\right) \phi_{ \pm}=0
$$

where for both (5.14) and (5.15), the KSE (5.10) and (5.12) are used to expand out the l.h.s. in terms of the fluxes. After some involved computation, making use of the field equations, one finds that (5.14) and (5.15) are in fact equivalent. This implies that there are no mixed integrability conditions between the $z$-direction and the rest. Therefore the independent KSEs to solve are (5.10) and (5.12).

Next, we consider the algebraic conditions (2.29) and (2.30). Note that these arise from the integrability conditions along the light-cone directions and they are not independent, i.e. they are implied by the remaining KSEs. Nevertheless, (2.29), (2.30), and together with (5.10) imply that

$$
\left(\partial_{z}\right)^{2} \phi_{ \pm} \pm \frac{1}{\ell} \partial_{z} \phi_{ \pm}=0
$$

This can be solved to yield

$$
\phi_{ \pm}=\sigma_{ \pm}+e^{\mp \frac{z}{\ell}} \tau_{ \pm}
$$

where

$$
\partial_{z} \sigma_{ \pm}=\partial_{z} \tau_{ \pm}=0
$$

and

$$
\Xi^{( \pm)} \sigma_{ \pm}=0, \quad \Xi^{( \pm)} \tau_{ \pm}=\mp \frac{1}{\ell} \tau_{ \pm} .
$$

To summarize, in order to find a solution to the KSEs for $A d S_{3} \times_{w} M^{8}$ backgrounds, it is sufficient to find spinors, $\tau_{ \pm}$and $\sigma_{ \pm}$which depend only on the coordinates of $M^{8}$ satisfying

$$
\begin{array}{rlrl}
D_{i}^{( \pm)} \sigma_{ \pm} & =0, & D_{i}^{( \pm)} \tau_{ \pm}=0, \\
\mathcal{A}^{( \pm)} \sigma_{ \pm}=0, & \mathcal{B}^{( \pm)} \tau_{ \pm}=0,
\end{array}
$$

where $D_{i}^{( \pm)}$is defined in (5.13) and

$$
\mathcal{A}^{( \pm)}=\Xi^{( \pm)}, \quad \mathcal{B}^{( \pm)}=\Xi^{( \pm)} \pm \frac{1}{\ell} .
$$

Having given a solution to (5.20), one can substitute it in (5.17) to construct the spinors $\phi_{ \pm}$. In turn, $\phi_{ \pm}$will solve the KSEs (2.26). Therefore to find the Killing spinors of the $A d S_{3} \times_{w} M^{8}$ backgrounds, it suffices to find the solutions of (5.20). 


\subsection{Counting supersymmetries}

Unlike for $A d S_{2}$ backgrounds, the counting of supersymmetries for $A d S_{3}$ backgrounds can be done using local geometry, and so additional requirements, like the conditions for the maximum principle to apply, are not necessary. For this we note that there is a 1-1 correspondence between the $\sigma_{-}$and $\sigma_{+}$solutions to the KSEs. Indeed given a $\sigma_{-}$solution of (5.20), then

$$
\sigma_{+}=A^{-1} \Gamma_{+} \Gamma_{z} \sigma_{-}
$$

automatically satisfies the KSEs, and conversely, if $\sigma_{+}$is a solution then so is

$$
\sigma_{-}=A \Gamma_{-} \Gamma_{z} \sigma_{+}
$$

There is also an identical 1-1 correspondence between $\tau_{+}$and $\tau_{-}$Killing spinors. Furthermore note that unlike for $A d S_{2}$ backgrounds, $\Theta_{-}$has a non-trivial kernel, i.e. $\Theta_{-} \tau_{-}=0$.

Using the relation between the $\left(\sigma_{-}, \tau_{-}\right)$and $\left(\sigma_{+}, \tau_{+}\right)$spinors described above, we conclude that the number of supersymmetries of the $A d S_{3}$ backgrounds is

$$
\begin{aligned}
N & =2\left(\operatorname{dim} \operatorname{Ker}\left(D^{(-)}, \mathcal{A}^{(-)}\right)+\operatorname{dim} \operatorname{Ker}\left(D^{(-)}, \mathcal{B}^{(-)}\right)\right), \\
& =2\left(\operatorname{dim} \operatorname{Ker}\left(D^{(+)}, \mathcal{A}^{(+)}\right)+\operatorname{dim} \operatorname{Ker}\left(D^{(+)}, \mathcal{B}^{(+)}\right)\right) .
\end{aligned}
$$

Therefore all such backgrounds preserve an even number of supersymmetries establishing (1.1).

\section{$6 \quad \mathrm{AdS}_{3}$ : global Analysis}

The number of supersymmetries preserved by $A d S_{3}$ backgrounds can also be counted from the zero modes of Dirac-like operators on $M^{8}$ as stated in (1.2). For this a 1-1 correspondence must be established between Killing spinors and the zero modes of these Dirac-like operators. The proof of this correspondence leads to new Lichnerowicz type theorems associated with the KSEs (5.20).

\subsection{A new Lichnerowicz theorem for $\tau_{+}$and $\sigma_{+}$}

One of the main difficulties in establishing (1.2) is to choose appropriate Dirac-like operators on $M^{8}$. The analysis is similar for $\sigma_{+}$and $\tau_{+}$spinors. Because of this, it is straightforward to describe both cases at once. Let $\chi$ denote either the $\sigma_{+}$or the $\tau_{+}$spinors and consider the operator

$$
\mathbb{D}_{i}^{(+)} \equiv D_{i}^{(+)}+k \Gamma_{i} \mathcal{C}^{(+)},
$$

where

$$
\mathcal{C}^{(+)}=\left(-\frac{1}{2} A^{-1} \not \partial A+\frac{1}{288} \not X+\frac{1}{6} A^{-1} \Gamma_{z} \not R+c A^{-1} \Gamma_{z}\right)
$$


$c$ is a constant, with $c=-\frac{1}{2 \ell}$ for $\chi=\sigma_{+}$and $c=\frac{1}{2 \ell}$ for $\chi=\tau_{+}$, and $k$ is another constant which will later be set to $k=-\frac{1}{8}$. Therefore $\mathcal{C}^{(+)}=\mathcal{A}^{(+)}$if the operator acts on $\sigma_{+}$, and $\mathcal{C}^{(+)}=\mathcal{B}^{(+)}$if the operator acts on $\tau_{+}$. Next observe that if $\chi$ is a Killing spinor, then $\mathbb{D}^{(+)} \chi=0$.

The associated Dirac equation to (6.1) is

$$
\begin{aligned}
\mathscr{D}^{(+)} \equiv \Gamma^{i} \mathbb{D}_{i}^{(+)} \chi=\Gamma^{i} D_{i}+( & \left(\frac{1}{2}-4 k\right) A^{-1} \not \partial A+\left(\frac{1}{72}+\frac{k}{36}\right) \not X \\
& \left.+\left(\frac{5}{12}+\frac{4}{3} k\right) A^{-1} \Gamma_{z} \emptyset+8 c k A^{-1} \Gamma_{z}\right) .
\end{aligned}
$$

In what follows, we shall demonstrate that the $\sigma_{+}, \tau_{+}$Killing spinors are in 1-1 correspondence with the zero modes of $\mathscr{D}^{(+)}$.

It is clear that if $\chi$ is a Killing spinor, then it is a zero mode of $\mathscr{D}^{(+)}$. It remains to prove the converse. For this assume that $\chi$ is a zero mode of $\mathscr{D}^{(+)}$, i.e. $\mathscr{D}^{(+)} \chi=0$, and that the fields of the theory satisfy the field equations and the Bianchi identities. Then we shall demonstrate that for $k=-\frac{1}{8}$

$$
D^{i} D_{i}\|\chi\|^{2}+3 A^{-1} D^{i} A D_{i}\|\chi\|^{2}=2\left\langle\mathbb{D}^{(+) i} \chi, \mathbb{D}_{i}^{(+)} \chi\right\rangle+\frac{9}{4}\left\|\mathcal{C}^{(+)} \chi\right\|^{2}
$$

An application of the maximum principle on the function $\|\chi\|^{2}$ reveals that the zero modes of $\mathscr{D}^{(+)}$are Killing spinors. Note that as we have demonstrated $A$ is nowhere zero, it follows that $A^{-1}$ is smooth if $A$ is smooth.

To show (6.4), we compute

$$
\begin{aligned}
D^{i} D_{i} \chi= & \Gamma^{k} D_{k}\left(\Gamma^{j} D_{j} \chi\right)+\frac{1}{4} R^{(8)} \chi \\
= & \Gamma^{k} D_{k}\left(\left(\left(4 k-\frac{1}{2}\right) A^{-1} \not \partial A-\left(\frac{1}{72}+\frac{k}{36}\right) \not X\right.\right. \\
& \left.\left.\quad-\left(\frac{5}{12}+\frac{4}{3} k\right) A^{-1} \Gamma_{z} \not-8 c k A^{-1} \Gamma_{z}\right) \chi\right)+\frac{1}{4} R^{(8)} \chi .
\end{aligned}
$$

One then obtains

$$
\begin{aligned}
\operatorname{Re}\left\langle\chi, D^{i} D_{i} \chi\right\rangle= & \left\langle\chi,\left(\left(-\frac{1}{2}+4 k\right) D^{k}\left(A^{-1} D_{k} A\right)+\frac{1}{4} \hat{R}+\left(\frac{5}{12}+\frac{4}{3} k\right) D^{k}\left(A^{-1} Q_{k}\right) \Gamma_{z}\right) \chi\right\rangle \\
& +\operatorname{Re}\left\langle\chi, \Gamma^{i}\left(\left(-\frac{1}{2}+4 k\right) A^{-1} \not \partial A-\left(\frac{1}{72}+\frac{k}{36}\right) \not X\right.\right. \\
& \left.\left.-\left(\frac{5}{12}+\frac{4}{3} k\right) A^{-1} \Gamma_{z} \not \subset-8 c k A^{-1} \Gamma_{z}\right) D_{i} \chi\right\rangle .
\end{aligned}
$$


We also write

$$
\begin{aligned}
\left\langle D^{i} \chi, D_{i} \chi\right\rangle= & \left\langle\mathbb{D}^{(+) i} \chi, \mathbb{D}_{i}^{(+)} \chi\right\rangle+\mathcal{F}_{1} \\
+ & \operatorname{Re}\left\langle\chi,\left(-A^{-1} D^{i} A+\frac{1}{144} \not X^{i}+\frac{1}{18} X^{i}\right.\right. \\
& -\frac{1}{6} A^{-1} \Gamma_{z} \not Q^{i}-\frac{1}{3} A^{-1} \Gamma_{z} Q^{i}+k\left(A^{-1} \not \partial A-\frac{1}{144} \not X\right. \\
& \left.\left.\left.+\frac{1}{3} A^{-1} \Gamma_{z} \not Q-2 c A^{-1} \Gamma_{z}\right) \Gamma^{i}\right) D_{i} \chi\right\rangle,
\end{aligned}
$$

where

$$
\begin{aligned}
& \mathcal{F}_{1}=\left\langle\chi,\left(-\frac{11}{32} A^{-2} D^{i} A D_{i} A+\frac{1}{384} A^{-1} D_{k_{1}} A \not X^{k_{1}}-\frac{5}{32} A^{-2} \Gamma_{z} Q_{i} D^{i} A\right.\right. \\
& +\frac{5}{24576} X_{k_{1} k_{2} k_{3} k_{4}} X_{k_{5} k_{6} k_{7} k_{8}} \Gamma^{k_{1} k_{2} k_{3} k_{4} k_{5} k_{6} k_{7} k_{8}}+\frac{1}{1024} X_{k_{1} k_{2} i j} X_{k_{3} k_{4}}{ }^{i j} \Gamma^{k_{1} k_{2} k_{3} k_{4}} \\
& -\frac{17}{3072} X^{2}-\frac{1}{128} A^{-1} \Gamma_{z} Q^{i} X_{i}-\frac{1}{16} A^{-2} Q^{2} \\
& \left.\left.+\frac{1}{384} c A^{-1} \Gamma_{z} X+\frac{1}{16} c A^{-2} \varnothing-\frac{1}{32 \ell^{2}} A^{-2}\right) \chi\right\rangle
\end{aligned}
$$

is a term which is purely algebraic in $Q, X, d A$ and $A$. We then evaluate

$$
\frac{1}{2} D^{i} D_{i}\|\chi\|^{2}=\operatorname{Re}\left\langle\chi, D^{i} D_{i} \chi\right\rangle+\left\langle D^{i} \chi, D_{i} \chi\right\rangle .
$$

We first consider the sum of the last two lines of (6.6) and the last three lines of (6.7). We require that this should be written in the form $\left\langle\chi, \mathcal{F}_{2} \Gamma^{k} D_{k} \chi\right\rangle$, where $\mathcal{F}_{2}$ is purely algebraic in $Q, X, d A$ and $A$. This imposes the condition $k=-\frac{1}{8}$ as was mentioned previously, with

$$
\mathcal{F}_{2}=-\frac{1}{384} \not X+\frac{7}{8} A^{-1} \not \supset A-\frac{1}{8} A^{-1} \Gamma_{z} \emptyset-\frac{3}{4} c A^{-1} \Gamma_{z} .
$$

If $k=-\frac{1}{8}$, one can then eliminate almost all of the conditions involving $D_{i} \chi$ by making use of the Dirac equation. However, there is one term of the type $\left\langle\chi, D^{k} A D_{k} \chi\right\rangle$, which cannot be entirely removed by such an elimination. This remaining term is equal to $-\frac{3}{2} A^{-1} D^{i} A D_{i}\|\chi\|^{2}$, and this will be retained.

We therefore set $k=-\frac{1}{8}$, and expand out the terms algebraic in the fluxes, making use of the bosonic field equations to eliminate the terms of the form $D^{i} X_{i k_{1} k_{2} k_{3}}, D^{i}\left(A^{-1} Q_{i}\right)$, $R^{(8)}$ and $D^{i} D_{i} A$ in terms of terms algebraic in the fluxes and $d A$. Then after some computation, we obtain (6.4).

To summarize, we have shown that for $k=-\frac{1}{8}$

$$
\mathscr{D}^{(+)} \chi=0 \Longleftrightarrow \mathbb{D}_{i}^{(+)} \chi=0, \quad \mathcal{C}^{(+)} \chi=0
$$

and furthermore

$$
\|\chi\|=\text { const }
$$


as a consequence of (6.4). In particular, if $\sigma_{+}, \tau_{+}$satisfy the Dirac equation (6.3) with $k=-\frac{1}{8}$, and $c=-\frac{1}{2 \ell}, c=\frac{1}{2 \ell}$, respectively, then $\sigma_{+}, \tau_{+}$satisfy the KSE (5.20), and furthermore

$$
\left\|\sigma_{+}\right\|=\text {const., } \quad\left\|\tau_{+}\right\|=\text {const. }
$$

This concludes the proof of a new Lichnerowicz type theorem for the connection (6.1).

\subsection{A new Lichnerowicz theorem for $\tau_{-}$and $\sigma_{-}$}

There is also an 1-1 correspondence between the $\tau_{-}, \sigma_{-}$Killing spinors and the zero modes of a Dirac-like operator on $M^{8}$. The proof follows from the relationship between $\tau_{-}, \sigma_{-}$ and $\tau_{+}, \sigma_{+}$spinors given in (5.22) and (5.23) and the corresponding relations for $\tau_{ \pm}$. Alteratively, one can repeat the analysis we have done for $\sigma_{+}$and $\tau_{+}$now for $\sigma_{-}$and $\tau_{-}$.

For this, we again treat both cases simultaneously by defining the operator

$$
\mathbb{D}_{i}^{(-)} \equiv D_{i}^{(-)} \psi+k \Gamma_{i} \mathcal{C}^{(-)},
$$

where

$$
\mathcal{C}^{(-)}=-\frac{1}{2} A^{-1} \not \partial A+\frac{1}{288} \not X-\frac{1}{6} A^{-1} \Gamma_{z} \not \subset+\tilde{c} A^{-1} \Gamma_{z} .
$$

The operator $\mathbb{D}^{(-)}$acts on the spinors $\psi$ such that for $\psi=\sigma_{-}$one has $\tilde{c}=\frac{1}{2 \ell}$, and for $\psi=\tau_{-}$one has $\tilde{c}=-\frac{1}{2 \ell}$. Equivalently, $\mathcal{C}^{(-)}=\mathcal{A}^{(-)}$for $\tilde{c}=\frac{1}{2 \ell}$ and $\mathcal{C}^{(-)}=\mathcal{B}^{(-)}$for $\tilde{c}=-\frac{1}{2 \ell}$. $k$ will be set to $k=-\frac{1}{8}$. It is clear from this that if $\psi$ is a Killing spinor, then $\mathbb{D}_{i}^{(-)} \psi=0$.

Next consider the Dirac-like operator

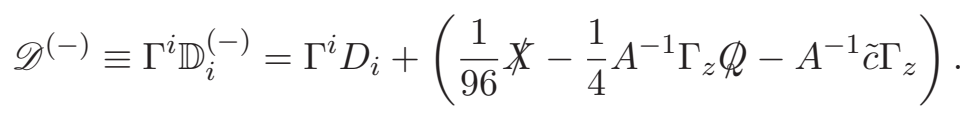

Observe that if $\psi$ is a Killing spinor, then $\psi$ is a zero mode of $\mathscr{D}^{(-)}$. To prove the converse, assume that $\mathscr{D}^{(-)} \psi=0$ and after performing a calculation similar to the one presented for $\mathscr{D}^{(+)}$, one can establish the formula

$$
D^{i} D_{i}\left(A^{-2}\|\psi\|^{2}\right)+3 A^{-1} D^{i} A D_{i}\left(A^{-2}\|\psi\|^{2}\right)=2 A^{-2}\left\langle\mathbb{D}^{(-) i} \psi, \mathbb{D}_{i}^{(-)} \psi\right\rangle+\frac{9}{4} A^{-2}\left\|\mathcal{C}^{(-)} \psi\right\|^{2},
$$

for $k=-\frac{1}{8}$. In fact, for the purely algebraic terms not obtained from the bosonic field equations and not involving $d A$, the resulting expressions are identical, modulo the replacements $Q \rightarrow-Q, c \rightarrow \tilde{c}$

It is clear that on applying the maximum principle to (6.17), it follows that

$$
\mathscr{D}^{(-)} \psi=0 \Longleftrightarrow \mathbb{D}_{i}^{(-)} \psi=0, \quad \mathcal{C}^{(-)} \psi=0,
$$

and furthermore that

$$
\left\|A^{-1} \psi\right\|=\text { const. }
$$


Therefore, if $\sigma_{-}, \tau_{-}$satisfy the Dirac equation (6.16) for $\tilde{c}=\frac{1}{2 \ell}$ and $\tilde{c}=-\frac{1}{2 \ell}$, respectively, then $\sigma_{-}, \tau_{-}$satisfy the KSEs (5.20), and furthermore

$$
\left\|A^{-1} \sigma_{-}\right\|=\text {const., } \quad\left\|A^{-1} \tau_{-}\right\|=\text {const. }
$$

This together with the similar result in the previous section establish the 1-1 correspondence between Killing spinors and zero modes of the Dirac-like operators.

\subsection{Counting supersymmetries again}

It is now straightforward to establish (1.2). Provided that the requirements for the validity of the new Lichnerowicz type theorems hold, the number of supersymmetries preserved by the $A d S_{3}$ backgrounds (5.24) can be rewritten as

$$
\begin{aligned}
N & =2\left(\operatorname{dim} \operatorname{Ker}\left(\mathscr{D}_{1 / 2 \ell}^{(-)}\right)+\operatorname{dim} \operatorname{Ker}\left(\mathscr{D}_{-1 / 2 \ell}^{(-)}\right)\right) \\
& =2\left(\operatorname{dim} \operatorname{Ker}\left(\mathscr{D}_{-1 / 2 \ell}^{(+)}\right)+\operatorname{dim} \operatorname{Ker}\left(\mathscr{D}_{1 / 2 \ell}^{(+)}\right)\right),
\end{aligned}
$$

where the subscripts denote the values of $\tilde{c}$ and $c$.

\section{$7 \quad \mathrm{AdS}_{4}$ : local analysis}

\subsection{Field equations}

The metric and fluxes induced on $\mathcal{S}=H^{2} \times{ }_{w} M^{7}$ from $A d S_{4} \times{ }_{w} M^{7}$ are

$$
\begin{aligned}
d \tilde{s}^{2} & =\left(\mathbf{e}^{z}\right)^{2}+\left(\mathbf{e}^{x}\right)^{2}+d s^{2}\left(M^{7}\right), & d s^{2}\left(M^{7}\right) & =\delta_{i j} \mathbf{e}^{i} \mathbf{e}^{j}, \\
Y & =S \mathbf{e}^{z} \wedge \mathbf{e}^{x}, & \tilde{F} & =X,
\end{aligned}
$$

where

$$
\mathbf{e}^{z}=A d z, \quad \mathbf{e}^{x}=A e^{z / \ell} d x .
$$

Substituting these into the Bianchi identities and field equations for $F$, we find that

$$
d X=0, \quad d\left(A^{4} S\right)=0,
$$

and

$$
D^{k} X_{k i_{1} i_{2} i_{3}}+4 D^{k} A X_{k i_{1} i_{2} i_{3}}=-\frac{1}{24} S \epsilon_{i_{1} i_{2} i_{3}}{ }^{k_{1} k_{2} k_{3} k_{4}} X_{k_{1} k_{2} k_{3} k_{4}},
$$

where we have set $d \operatorname{vol}(\mathcal{S})=\mathbf{e}^{z} \wedge \mathbf{e}^{x} \wedge d \operatorname{vol}\left(M^{7}\right)$.

Furthermore, the Einstein equations reduce on $M^{7}$ as

$$
D^{k} D_{k} \log A=-\frac{3 A^{-2}}{\ell^{2}}-4 \partial_{k} \log A \partial^{k} \log A+\frac{1}{3} S^{2}+\frac{1}{144} X^{2},
$$

and

$$
R_{i j}^{(7)}-4 D_{i} \partial_{j} \log A-4 \partial_{i} \log A \partial_{j} \log A=\frac{1}{12} X_{i j}^{2}+\delta_{i j}\left(\frac{1}{6} S^{2}-\frac{1}{114} X^{2}\right),
$$

where $R^{(7)}$ is the Ricci tensor on $M^{7}$. This completes the reduction of the Bianchi identities and field equations on $M^{7}$. 


\subsubsection{The warp factor $A$ is nowhere vanishing}

Using a similar argument based of the field equations as that in the $A d S_{3}$ case, one can again demonstrate that $A$ is nowhere vanishing on $M^{7}$. Again $A^{2}$ is related via the KSEs to the length of a parallel spinor confirming that it cannot vanish anywhere.

\subsection{Integrability of KSEs along AdS}

To integrate the independent KSEs (2.26) along the remaining AdS directions, we shall first integrate along $\mathbf{e}^{z}$ and then the remaining $\mathbf{e}^{x}$ direction. The integration along the $\mathbf{e}^{z}$ direction proceeds as in the previous $A d S_{3} \times_{w} M^{8}$ backgrounds and after using (2.29) and (2.30), we find that

$$
\phi_{ \pm}=\eta_{ \pm}+e^{\mp z / \ell} \chi_{ \pm}
$$

where

$$
\Xi^{( \pm)} \eta_{ \pm}=0, \quad \Xi^{ \pm} \chi_{ \pm}=\mp \frac{1}{\ell} \chi_{ \pm}
$$

and

$$
\Xi^{( \pm)}=-\frac{1}{2} \Gamma_{z} \not \partial A \mp \frac{1}{2 \ell}+\frac{1}{288} \Gamma_{z} A X \pm \frac{1}{6} A S \Gamma_{x} .
$$

The spinors $\eta_{ \pm}$and $\chi_{ \pm}$depend on the $x$ coordinate of $A d S_{4}$ and the coordinates of $M^{7}$. Next we integrate along the $x$ coordinate to find that

$$
\phi_{+}=\sigma_{+}-\frac{1}{\ell} x \Gamma_{x} \Gamma_{z} \tau_{+}+e^{-z / \ell} \tau_{+}, \quad \phi_{-}=\sigma_{-}+e^{z / \ell}\left(-\frac{1}{\ell} x \Gamma_{x} \Gamma_{z} \sigma_{-}+\tau_{-}\right)
$$

where $\sigma_{ \pm}$and $\tau_{ \pm}$depend only on the coordinates of $M^{7}$. There are no further conditions to consider.

To summarize after integration over all AdS directions, the independent KSEs are

$$
D_{i}^{( \pm)} \sigma_{ \pm}=0, \quad D_{i}^{( \pm)} \tau_{ \pm}=0,
$$

and

$$
\mathcal{A}^{( \pm)} \sigma_{ \pm}=0, \quad \mathcal{B}^{( \pm)} \tau_{ \pm}=0
$$

where

$$
\begin{aligned}
& D_{i}^{( \pm)} \equiv D_{i} \pm \frac{1}{2} \partial_{i} \log A-\frac{1}{288} \not X_{i}+\frac{1}{36} X_{i} \pm \frac{1}{12} S \Gamma_{i z x} \\
& \mathcal{A}^{( \pm)} \equiv \Xi^{( \pm)}, \quad \mathcal{B}^{( \pm)} \equiv \Xi^{( \pm)} \pm \frac{1}{\ell}
\end{aligned}
$$

Therefore, the KSEs reduce to a set of parallel transport and algebraic equations on the transverse spaces $M^{7}$. 


\subsection{Counting supersymmetries}

To find the supersymmetries preserved by the $A d S_{4} \times{ }_{w} M^{7}$ backgrounds, notice that if $\sigma_{ \pm}$ is a solution of the KSEs, then

$$
\tau_{ \pm}=\Gamma_{z x} \sigma_{ \pm}
$$

is also a solution, and vice versa as $\Gamma_{z x}$ is invertible. Furthermore, observe that if $\sigma_{-}, \tau_{-}$ is a solution, so is

$$
\sigma_{+}=A^{-1} \Gamma_{+} \Gamma_{z} \sigma_{-}, \quad \tau_{+}=A^{-1} \Gamma_{+} \Gamma_{z} \tau_{-} .
$$

and similarly, if $\sigma_{+}, \tau_{+}$is a solution, so is

$$
\sigma_{-}=A \Gamma_{-} \Gamma_{z} \sigma_{+}, \quad \tau_{-}=A \Gamma_{-} \Gamma_{z} \tau_{+} .
$$

From the above relations, one concludes that the $A d S_{4} \times_{w} M^{7}$ backgrounds preserve

$$
N=4 \operatorname{dimKer}\left(D^{(-)}, \mathcal{A}^{(-)}\right)
$$

supersymmetries for $0<\operatorname{dimKer}\left(D^{(-)}, \mathcal{A}^{(-)}\right) \leq 8$. This proves $(1.1)$. For $\operatorname{dim} \operatorname{Ker}\left(D^{(-)}\right.$, $\left.\mathcal{A}^{(-)}\right)=8$, there is a unique solution which is locally isometric to $A d S_{4} \times S^{7}$.

\section{$8 \quad \mathrm{AdS}_{4}$ : global analysis}

\subsection{A new Lichnerowicz theorem for $\tau_{+}$and $\sigma_{+}$}

To prove (1.2), one has to establish a 1-1 correspondence between Killing spinors and zero modes of a Dirac-like operator. To identify the appropriate Dirac-like operator, as for the $A d S_{3}$ case, we take a linear combination of the (7.11) and (7.12) KSEs and consider the modified gravitino KSE operator

$$
\mathbb{D}_{i}^{(+)} \equiv D_{i}^{(+)}+k \Gamma_{i} \mathcal{C}^{(+)},
$$

where

$$
\mathcal{C}^{(+)}=-\frac{1}{2} A^{-1} \Gamma^{k} D_{k} A+\frac{1}{288} \not X+\frac{1}{6} S \Gamma_{z x}+c A^{-1} \Gamma_{z} .
$$

$\mathbb{D}^{(+)}$is acting on the spinors $\chi$ such that for $\chi=\sigma_{+}$, one has $c=-\frac{1}{2 \ell}$, and for $c=\frac{1}{2 \ell}$, one has $\chi=\tau_{+}$. In what follows, one determines $k$ to formulate a maximum principle for the length square $\|\chi\|^{2}$ of the spinor $\chi$.

The associated Dirac equation to (8.1) is

$$
\mathscr{D}^{(+)} \equiv \Gamma^{i} \mathbb{D}_{i}^{(+)}=\Gamma^{i} D_{i}+\left[\frac{1-7 k}{2} A^{-1} \not \partial A+\frac{5+7 k}{288} \not X+\frac{7}{4} S \Gamma_{z x}+7 c k A^{-1} \Gamma_{z}\right] .
$$

Assuming now that $\mathscr{D}^{(+)} \chi=0$ and using the Bianchi identities and field equations, one can prove following steps similar to those described for the $A d S_{3}$ case that

$$
D^{2}\|\chi\|^{2}+4 A^{-1} \partial^{i} A \partial_{i}\|\chi\|^{2}=2\left\langle\mathbb{D}_{i}^{(+)} \chi, \mathbb{D}^{(+) i} \chi\right\rangle+\frac{36}{7}\left\|\mathcal{C}^{(+)} \chi\right\|^{2},
$$

provided that $k=-2 / 7$. 
Applying the maximum principle on $\|\chi\|^{2}$, one finds that

$$
\mathscr{D}^{(+)} \chi=0 \Longleftrightarrow \mathbb{D}_{i}^{(+)} \chi=0, \quad \mathcal{C}^{(+)} \chi=0
$$

and in addition

$$
\|\chi\|=\text { const }
$$

This establishes a 1-1 correspondence between the $\sigma_{+}$and $\tau_{+}$Killing spinors and zero modes of $\mathscr{D}^{(+)}$.

\subsection{A new Lichnerowicz theorem for $\tau_{-}$and $\sigma_{-}$}

A Lichnerowicz type theorem can be proven for the $\tau_{-}$and $\sigma_{-}$Killing spinors following similar steps to those described in the previous section. Alternatively, one can use the relation between $\tau_{-}, \sigma_{-}$and $\tau_{+}, \sigma_{+}$spinors described in (7.15) and (7.16). In either case, assuming that $\psi$ is a zero mode of a modified Dirac-like operator $\mathscr{D}^{(-)}$, one can establish the equality

$$
D^{2}\left\|A^{-1} \psi\right\|^{2}+4 A^{-1} \partial^{i} A \partial_{i}\left\|A^{-1} \psi\right\|^{2}=2 A^{-2}\left\langle\mathbb{D}_{i}^{(-)} \psi, \mathbb{D}^{(-) i} \psi\right\rangle+\frac{36}{7} A^{-2}\left\|\mathcal{C}^{(-)} \psi\right\|^{2},
$$

where $\mathbb{D}^{(-)}=D^{(-)}+k \Gamma_{i} \mathcal{C}^{(-)}$is the modified gravitino KSE operator,

$$
\mathcal{C}^{(-)}=-\frac{1}{2} A^{-1} \not \partial A+\tilde{c} A^{-1} \Gamma_{z}+\frac{1}{288} \not X-\frac{1}{6} S \Gamma_{z x},
$$

$k=-2 / 7$ and $\tilde{c}=\frac{1}{2 \ell}$ for $\psi=\sigma_{-}$and $\tilde{c}=-\frac{1}{2 \ell}$ for $\psi=\tau_{-}$. Moreover $\mathscr{D}^{(-)}=\Gamma^{i} \mathbb{D}_{i}^{(-)}$. An application of the maximum principle implies that

$$
\mathscr{D}^{(-)} \psi=0 \Longleftrightarrow \mathbb{D}_{i}^{(-)} \psi=0, \quad \mathcal{C}^{(-)} \psi=0
$$

and that

$$
\left\|A^{-1} \psi\right\|=\text { const } .
$$

The warp factor is therefore related to the length of the Killing spinors. Combining the results of this with the previous section, we have established a 1-1 correspondence between the Killing spinors and the zero modes of Dirac-like operators on $M^{7}$.

\subsection{Counting supersymmetries}

Now we can establish (1.2). It is simply a consequence of the Lichnerowicz type theorems proved in the previous two sections. In particular, the number of supersymmetries preserved by $A d S_{4} \times{ }_{w} M^{7}$ backgrounds (7.17) can now be written as

$$
N=4 \operatorname{dim} \operatorname{Ker} \mathscr{D}_{1 / 2 \ell}^{(-)} .
$$

This formula can also be expressed in terms of any other three choices of Dirac-like operators. 


\section{$9 \quad \operatorname{AdS}_{n} n>4$ : local analysis}

\subsection{Bianchi identities and field equations}

The metric and fluxes induced on $\mathcal{S}=H^{n-2} \times{ }_{w} M^{11-n}$ from $A d S_{n} \times{ }_{w} M^{11-n}, n>4$, are

$$
\begin{aligned}
d \tilde{s}^{2} & =\left(\mathbf{e}^{z}\right)^{2}+\delta_{\mathrm{ab}} \mathbf{e}^{\mathrm{a}} \mathbf{e}^{\mathrm{b}}+d s^{2}\left(M^{11-n}\right), \quad d s^{2}\left(M^{11-n}\right)=\delta_{i j} \mathbf{e}^{i} \mathbf{e}^{j}, \\
\tilde{F} & =X,
\end{aligned}
$$

where

$$
\mathbf{e}^{z}=A d z, \quad \mathbf{e}^{\mathrm{a}}=A e^{z / \ell} d x^{a} .
$$

The Bianchi identities and field equations of the fluxes can be written as

$$
D^{k} X_{k i_{1} i_{2} i_{3}}=-n A^{-1} D^{k} A X_{k i_{1} i_{2} i_{3}}, \quad d X=0 .
$$

Similarly after a decomposition, the independent Einstein field equations are

$$
D^{k} \partial_{k} \log A=-\frac{n-1}{\ell^{2}} A^{-2}-n \partial^{k} \log A \partial_{k} \log A+\frac{1}{144} X^{2},
$$

and

$$
R_{i j}^{(11-n)}-n D_{i} \partial_{j} \log A-n \partial_{i} \log A \partial_{j} \log A=\frac{1}{12} X_{i j}^{2}-\frac{1}{144} \delta_{i j} X^{2}
$$

This completes the decomposition of Bianchi and the field equations on $M^{11-n}$.

\subsubsection{The warp factor $A$ is nowhere vanishing}

For $A d S_{n} \times{ }_{w} M^{11-n}$ backgrounds, the warp factor does not vanish at any point of $M^{11-n}$. This follows from the field equations as in all the previous cases.

\subsection{Integrability of KSEs along AdS}

To integrate the independent KSEs (2.26) along the remaining AdS directions, we shall first integrate along $\mathbf{e}^{z}$ and then the remaining $\mathbf{e}^{\mathrm{a}}$ directions. The integration along the $\mathbf{e}^{z}$ direction proceeds as in the previous examples and after using (2.29) and (2.30, we find that

$$
\phi_{ \pm}=\eta_{ \pm}+e^{\mp z / \ell} \chi_{ \pm}
$$

where

$$
\Xi^{( \pm)} \eta_{ \pm}=0, \quad \Xi^{( \pm)} \chi_{ \pm}=\mp \frac{1}{\ell} \chi_{ \pm}
$$

and

$$
\Xi^{( \pm)}=-\frac{1}{2} \Gamma_{z} \not \partial \mp \mp \frac{1}{2 \ell}+\frac{1}{288} \Gamma_{z} A X
$$


The spinors $\eta_{ \pm}$and $\chi_{ \pm}$depend on the $x^{a}$ and the coordinates of $M$. Next we integrate along the $x^{a}$ coordinates to find that

$$
\phi_{+}=\sigma_{+}-\frac{1}{\ell} x^{a} \Gamma_{\mathrm{a}} \Gamma_{z} \tau_{+}+e^{-z / \ell} \tau_{+}, \quad \phi_{-}=\sigma_{-}+e^{z / \ell}\left(-\frac{1}{\ell} x^{a} \Gamma_{\mathrm{a}} \Gamma_{z} \sigma_{-}+\tau_{-}\right),
$$

where $\sigma_{ \pm}$and $\tau_{ \pm}$depend only on the coordinates of $M^{11-n}$.

Furthermore after integration over all AdS directions, the independent KSEs are

$$
D_{i}^{( \pm)} \sigma_{ \pm}=0, \quad D_{i}^{( \pm)} \tau_{ \pm}=0
$$

and

$$
\mathcal{A}^{( \pm)} \sigma_{ \pm}=0, \quad \mathcal{B}^{( \pm)} \tau_{ \pm}=0
$$

where

$$
\begin{aligned}
& D_{i}^{( \pm)}=D_{i} \pm \frac{1}{2} \partial_{i} \log A-\frac{1}{288} \not X_{i}+\frac{1}{36} X_{i}, \\
& \mathcal{A}^{( \pm)}=\Xi^{( \pm)}, \quad \mathcal{B}^{( \pm)}=\Xi^{( \pm)} \pm \frac{1}{\ell}
\end{aligned}
$$

Therefore, the investigation of solutions to the KSEs reduces to a set of parallel transport and algebraic equations on the transverse spaces $M^{11-n}$.

\subsection{Counting supersymmetries}

To find the supersymmetries preserved by the $A d S_{n} \times{ }_{w} M^{11-n}$ backgrounds, notice that if $\sigma_{ \pm}$is a solution of the KSEs, then

$$
\tau_{ \pm}(v)=v^{\mathrm{a}} \Gamma_{z} \Gamma_{\mathrm{a}} \sigma_{ \pm}
$$

is also a solution for any constant vector $v$, and vice versa. In addition if two vectors $v$ are orthogonal, then the associated spinors $\tau_{ \pm}(v)$ are also orthogonal and so linearly independent. Furthermore, observe that if $\sigma_{-}, \tau_{-}$is a solution, so is

$$
\sigma_{+}=A^{-1} \Gamma_{+} \Gamma_{z} \sigma_{-}, \quad \tau_{+}=A^{-1} \Gamma_{+} \Gamma_{z} \tau_{-} .
$$

Similarly, if $\sigma_{+}, \tau_{+}$is a solution, so is

$$
\sigma_{-}=A \Gamma_{-} \Gamma_{z} \sigma_{+}, \quad \tau_{-}=A \Gamma_{-} \Gamma_{z} \tau_{+} .
$$

As a result if $\sigma_{-}$is Killing spinor, then

$$
\Gamma_{\mathrm{ab}} \sigma_{-}, \quad \mathrm{a}<\mathrm{b},
$$

are also Killing spinors of the same KSEs, i.e. $D^{(-)} \Gamma_{\mathrm{ab}} \sigma_{-}=\Xi^{(-)} \Gamma_{\mathrm{ab}} \sigma_{-}=0$. However not all $\left(\sigma_{-}, \Gamma_{\mathrm{ab}} \sigma_{-}\right), \mathrm{a}<\mathrm{b}$, are linearly independent. Clearly if $\left\{\sigma_{-}, \Gamma_{\mathrm{ab}} \sigma_{-}\right\}, \mathrm{a}<\mathrm{b}$ are mutually orthogonal, they are linearly independent. $\sigma_{-}$is orthogonal to all $\Gamma_{\mathrm{ab}} \sigma_{-}$, and $\Gamma_{\mathrm{ab}} \sigma_{-}$is orthogonal to $\Gamma_{\mathrm{a}^{\prime} \mathrm{b}^{\prime}} \sigma_{-}$, iff the bilinear

$$
\left\langle\sigma_{-}, \Gamma_{\mathrm{ab}} \Gamma_{\mathrm{a}^{\prime} \mathrm{b}^{\prime}} \sigma_{-}\right\rangle=0 .
$$


Using these, one can count the number of supersymmetries preserved by $A d S_{n} \times{ }_{w} M^{11-n}$ backgrounds. A straightforward analysis reveals that if the solutions exist, $A d S_{5} \times_{w} M^{6}$ backgrounds preserve $8 k$ supersymmetries and $A d S_{6} \times_{w} M^{5}$ backgrounds preserve $16 k$ supersymmetries. Note that

$$
\operatorname{dim} \operatorname{Ker}\left(D^{(-)}, \Xi^{(-)}\right)=2 k, \quad \operatorname{dim} \operatorname{Ker}\left(D^{(-)}, \Xi^{(-)}\right)=4 k,
$$

respectively. In these two cases, the orthogonality condition (9.17) is always automatically satisfied.

For $A d S_{7} \times{ }_{w} M^{4},(9.17)$ gives a non-trivial restriction for $\mathrm{a}, \mathrm{b}, \mathrm{a}^{\prime}$ and $\mathrm{b}^{\prime}$ distinct. To see this observe that $\Gamma_{\text {aba' }} \mathrm{b}^{\prime}$ commutes with the KSEs $\left(D^{(-)}, \Xi^{(-)}\right)$and so $\sigma_{-}$can be taken to be in one of the two eigenspaces of $\Gamma_{\mathrm{aba} \mathrm{b}^{\prime}}$ with eigenvalues \pm 1 . For $\sigma_{-}$in one of the two eigenspaces, only 4 from the 7 spinors $\left\{\sigma_{-}, \Gamma_{\mathrm{ab}} \sigma_{-}\right\}, \mathrm{a}<\mathrm{b}$, are linearly independent. As a result $A d S_{7} \times{ }_{w} M^{4}$ can preserve $16 k$ supersymmetries with $k$ given by the second equation in (9.18).

To summarize, we have the following:

(i) $A d S_{5} \times_{w} M^{6}$ backgrounds can preserve 8, 16 and 24 supersymmetries. This follows from the results of [16] which rule out the existence of such backgrounds preserving 32 supersymmetries. It also turns out that there are no $A d S_{5} \times_{w} M^{6}$ preserving 24 supersymmetries. The proof of this will be demonstrated in [15].

(ii) $A d S_{6} \times_{w} M^{5}$ backgrounds can preserve 16 supersymmetries. The existence of such backgrounds preserving 32 supersymmetries is ruled out from the results of [16]. It turns out that under some assumptions there are no backgrounds preserving 16 supersymmetries either, and a proof will be presented in [15], see also [14].

(iii) $A d S_{7} \times{ }_{w} M^{4}$ can preserve 16 and 32 supersymmetries with the latter being locally isometric to the maximally supersymmetric $A d S_{7} \times S^{4}$ background of 11-dimensional supergravity. In fact all $A d S_{7} \times_{w} M^{4}$ solutions are locally isometric to $A d S_{7} \times S^{4}$. This follows from the results of [28] mentioned in [8].

Collecting all these results together, we establish (1.1) for the rest of the $A d S_{n}$ backgrounds.

\section{$10 \quad \operatorname{AdS}_{n} n>4$ : global analysis}

In this section, we prove (1.2). For this, we shall demonstrate new Lichnerowicz type theorems associated with the KSEs (9.10) and (9.11).

\subsection{A new Lichnerowicz Theorem for $\tau_{+}$and $\sigma_{+}$}

To show that there is a 1-1 correspondence between Killing spinors and zero modes of Diraclike operators, we take a linear combination of the (9.10) and (9.11) KSEs and consider the modified gravitino KSE operator

$$
\mathbb{D}_{i}^{(+)} \equiv D_{i}^{(+)}+k \Gamma_{i} \mathcal{C}^{(+)},
$$


where

$$
\mathcal{C}^{(+)}=-\frac{1}{2} A^{-1} \Gamma^{k} D_{k} A+\frac{1}{288} \not X+c A^{-1} \Gamma_{z} .
$$

This operator acts on spinors $\chi$ such that for $\chi=\sigma_{+}, c=-\frac{1}{2 \ell}$, and for $\chi=\tau_{+}, c=\frac{1}{2 \ell}$. The associated Dirac equation to (10.1) is

$$
\mathscr{D}^{(+)} \equiv \Gamma^{i} D_{i}+\left[\frac{1-k(11-n)}{2} A^{-1} \not \partial A+\frac{n+1+k(11-n)}{288} \not X+c k(11-n) A^{-1} \Gamma_{z}\right] .
$$

Assuming now that $\mathscr{D}^{(+)} \chi=0$ and using the Bianchi identities and field equations, one can prove following steps similar to those described for the $A d S_{3}$ backgrounds that

$$
D^{2}\|\chi\|^{2}+n A^{-1} \partial^{i} A \partial_{i}\|\chi\|^{2}=2\left\langle\mathbb{D}_{i}^{(+)} \chi, \mathbb{D}^{(+) i} \chi\right\rangle+2 \frac{9 n-18}{11-n}\left\|\mathcal{C}^{(+)} \chi\right\|^{2},
$$

provided that $k=2-n / 11-n$.

For completeness, one has that

$$
\begin{aligned}
\mathcal{F}_{1}=\langle\chi,[ & -\frac{11-3 n+n^{2}}{4(11-n)}(d \log A)^{2}+\frac{15-3 n}{288(11-n)} \partial_{k} \log A \not K X^{k} \\
& +\frac{63-18 n}{1152(11-n)} X_{i j k_{1} k_{2}} X^{i j}{ }_{k_{3} k_{4}} \Gamma^{k_{1} k_{2} k_{3} k_{4}} \\
& \left.\left.+\frac{-261+36 n}{3456(11-n)} X^{2}+\frac{3 n-6}{144(11-n)} c A^{-1} \Gamma_{z} X-\frac{(2-n)^{2}}{4(11-n)} A^{-2}\right] \chi\right\rangle
\end{aligned}
$$

and

$$
\mathcal{F}_{2}=\frac{10 n-n^{2}-7}{2(11-n)} A^{-1} \not \partial A+\frac{-15+3 n}{288(11-n)} \not X+\frac{(2-n)(9-n)}{11-n} c A^{-1} \Gamma_{z} .
$$

Applying the maximum principle one concludes that

$$
\mathscr{D}^{(+)} \chi=0 \Longleftrightarrow \mathbb{D}_{i}^{(+)} \chi=0, \quad \mathcal{C}^{(+)} \chi=0
$$

and that

$$
\|\chi\|=\text { const }
$$

This proves the correspondence between the Killing spinors $\sigma_{+}$and $\tau_{+}$and the zero modes of $\mathscr{D}^{(+)}$.

\subsection{A new Lichnerowicz Theorem for $\tau_{-}$and $\sigma_{-}$}

The new Lichnerowicz theorem on the $\tau_{-}$and $\sigma_{-}$can be formulated either by following a calculation similar to that of the previous section or more simply by utilizing the relationship between the positive and negative lightcone chirality spinors and their associated KSEs given in (9.14) and (9.15). The maximum principle formula can be written as

$D^{2}\left\|A^{-1} \psi\right\|^{2}+n A^{-1} \partial^{i} A \partial_{i}\left\|A^{-1} \psi\right\|^{2}=2 A^{-2}\left\langle\mathbb{D}_{i}^{(-)} \psi, \mathbb{D}^{(-) i} \psi\right\rangle+2 \frac{9 n-18}{11-n} A^{-2}\left\|\mathcal{C}^{(-)} \psi\right\|^{2}$, 


\begin{tabular}{|c|c|}
\hline$A d S_{n} \times_{w} M^{11-n}$ & $N$ \\
\hline$n=2$ & $2 k, k<15$ \\
\hline$n=3$ & $2 k, k<15$ \\
\hline$n=4$ & $4 k, k \leq 8$ \\
\hline$n=5$ & $8,16,24$ \\
\hline$n=6$ & 16 \\
\hline$n=7$ & 16,32 \\
\hline
\end{tabular}

Table 1. The number of supersymmetries $N$ of $A d S_{n} \times_{w} M$ backgrounds are given. For $A d S_{2} \times{ }_{w} M^{9}$, one can show that these backgrounds preserve an even number of supersymmetries provided that they are smooth and $M^{9}$ is compact without boundary. For the rest, the counting of supersymmetries does not rely on the compactness of $M^{11-n}$. The bounds in $k$ arise from the non-existence of supersymmetric solutions with near maximal supersymmetry. For the remaining fractions, it is not known whether there always exist backgrounds preserving the prescribed number of supersymmetries.

where

$$
\begin{aligned}
& \mathbb{D}_{i}^{(-)}=D_{i}^{(-)}+k \Gamma_{i} \mathcal{C}^{(-)}, \\
& \mathcal{C}^{(-)}=-\frac{1}{2} A^{-1} \not \partial A+\tilde{c} A^{-1} \Gamma_{z}+\frac{1}{288} \not X,
\end{aligned}
$$

is the modified gravitino KSE, $k=2-n / 11-n$ and $\tilde{c}=\frac{1}{2 \ell}$ for $\psi=\sigma_{-}$and $\tilde{c}=-\frac{1}{2 \ell}$ for $\psi=\tau_{-}$. The application of the maximum principle implies that

$$
\mathscr{D}^{(-)} \psi=0 \Longleftrightarrow \mathbb{D}_{i}^{(-)} \psi=0, \quad \mathcal{C}^{(-)} \psi=0
$$

and that

$$
\left\|A^{-1} \psi\right\|=\text { const } .
$$

Thus, the warp factor is proportional to the length of the Killing spinor.

\subsection{Counting supersymmetries}

It is now straightforward to prove (1.2) for $A d S_{n} \times{ }_{w} M^{11-n}, n>4$ backgrounds. In particular this follows from (1.1) and after setting

$$
N_{-}=\operatorname{dim} \operatorname{Ker} \mathscr{D}_{1 / 2 \ell}^{(-)} .
$$

This completes the proof of (1.2) for all $A d S_{n}$ backgrounds.

\section{Do the Killing spinors factorize?}

In many of the investigations of $A d S_{n}$ solutions of supergravity theories, it is assumed that the Killing spinors of the spacetime factorize as

$$
\epsilon=\xi \otimes \psi
$$


where $\xi$ is a Killing spinor on $A d S_{n}$ satisfying the Killing spinor equation

$$
\nabla_{\mu} \xi+\lambda \gamma_{\mu} \xi=0
$$

and $\psi$ is a spinor on the transverse space, and where $\mu$ are $A d S_{n}$ indices and $\gamma_{\mu}$ are $A d S_{n}$ gamma matrices. Then the ansatz (11.1) is substituted into the KSEs to derive the equations that must be satisfied by $\psi$. This factorization has been instrumental in many of the AdS computations. Here we shall examine whether the Killing spinors can always be written in this way.

It is not straightforward to adapt our results to the ansatz (11.1) for the spinors stated above. This is because of the ambiguities that one encounters when decomposing the spacetime gamma matrices in terms of those of AdS and transverse space as well as the differences in the choice of coordinates on $A d S_{n}$ and spacetime frame. However we can restrict our Killing spinors on $A d S_{n}$ and examine whether they satisfy (11.2). Indeed, if the Killing spinors factorize as in (11.1) and $\psi$ is taken to be a constant, then $\epsilon$ solves (11.2).

Consider first the $A d S_{2}$ case. Observe that after using the integrability condition (2.30) the Killing spinors can be written as

$$
\epsilon=\phi_{+}+\phi_{-}+u \Gamma_{+} \Theta_{-} \phi_{-}+r \Gamma_{-} \Theta_{+} \phi_{+}+\frac{\Delta}{2} r u \phi_{-},
$$

where $\phi_{ \pm}$lie in the 16 Majorana representation of $\operatorname{Spin}(9)$, the spin group of $M^{9}$, and they are localized on $M^{9}$. $\phi_{ \pm}$are the candidates to be identified with $\psi$. On imposing the condition (11.2) we find

$$
\left(\Theta_{ \pm}+\lambda\right) \phi_{ \pm}=0, \quad \lambda^{2}=\frac{\Delta}{4}
$$

However, these conditions are incompatible with the KSEs. To see this, observe that the horizon Dirac equations on $\mathcal{S}$ can be written as

$$
\Gamma^{i} \tilde{\nabla}_{i} \psi_{ \pm}+3 \Theta_{ \pm} \psi_{ \pm}=0
$$

where $\psi_{+}=\Delta^{-1} \phi_{+}, \psi_{-}=\Delta^{-\frac{1}{2}} \phi_{-}$. Then (11.5) implies that

$$
\int_{M^{9}}\left\langle\psi_{ \pm}, \Theta_{ \pm} \psi_{ \pm}\right\rangle=0
$$

However, substituting (11.4) into (11.6) then leads to a contradiction. Hence the $A d S_{2}$ background spinors cannot factorize as in (11.1).

Next let us turn to the $A d S_{3}$ case. After applying the integrability condition (2.30) and using the algebraic KSEs (5.19), the Killing spinor can be written as

$$
\epsilon=\sigma_{+}+\sigma_{-}+e^{-\frac{z}{\ell}} \tau_{+}+e^{\frac{z}{\ell}} \tau_{-}-\frac{u}{\ell} A^{-1} \Gamma_{+z} \sigma_{-}-\frac{r}{\ell} A^{-1} e^{-\frac{z}{\ell}} \Gamma_{-z} \tau_{+} .
$$

Observe that $\sigma_{ \pm}$and $\tau_{ \pm}$are in the $\mathbf{1 6}$ Majorana (but not Weyl) representation of $\operatorname{Spin}(8)$, the spin group of $M^{8}$, and they are localized on $M^{8}$. Next let us restrict $\epsilon$ on $A d S_{3}$ by treating $\sigma_{ \pm}$and $\tau_{ \pm}$as integration constants, i.e. suppress all the dependence of $\epsilon$ on the 
coordinates of $M^{8}$, and investigate whether the resulting spinor solves the KSE (11.2) on $A d S_{n}$ for some choice of $\lambda$. Substituting $\epsilon$ into (11.2), one finds that it solves the KSE if and only if

$$
\frac{1}{2 \ell} A^{-1} \Gamma_{z} \sigma_{ \pm} \pm \lambda \sigma_{ \pm}=0, \quad \frac{1}{2 \ell} A^{-1} \Gamma_{z} \tau_{ \pm} \mp \lambda \tau_{ \pm}=0
$$

Let us focus on the condition (11.8) for $\sigma_{+}$. This condition can be solved by imposing the projection $\Gamma_{z} \sigma_{+}^{ \pm}= \pm \sigma_{+}^{ \pm}$with $\sigma_{+}=\sigma_{+}^{+}+\sigma_{+}^{-}$and $\lambda=\mp \frac{1}{2 \ell} A^{-1}$. Therefore $\epsilon$ solves (11.2) if and only if one of the components of $\sigma_{+}, \sigma_{+}^{+}$or $\sigma_{+}^{-}$, is set to zero. However, this is an additional condition on $\sigma_{+}$which does not arise from the KSEs as applied to $A d S_{3}$ backgrounds. In fact since $\Gamma_{z}$ does not commute with the KSEs, one cannot consistently set $\sigma_{+}^{+}$or $\sigma_{+}^{-}$to zero without imposing an additional restriction on the fields. A similar analysis can be done for the remaining three cases. Therefore for generic $A d S_{3}$ backgrounds, the Killing spinors cannot be written as in (11.1) satisfying (11.2).

Next let us consider the same question for $A d S_{n}$ backgrounds for $n>3$. The Killing spinors for these backgrounds, after using the integrability condition (2.30) and the algebraic KSEs $\mathcal{A}^{( \pm)} \sigma_{ \pm}=\mathcal{B}^{( \pm)} \tau_{ \pm}=0$, can be written as

$$
\begin{aligned}
\epsilon= & \sigma_{+}+\sigma_{-}+e^{-\frac{z}{\ell}} \tau_{+}+e^{\frac{z}{\ell}} \tau_{-} \\
& -\frac{1}{\ell}\left(u A^{-1} \Gamma_{+z} \sigma_{-}+r A^{-1} e^{-\frac{z}{\ell}} \Gamma_{-z} \tau_{+}+\sum_{\mathrm{a}} x^{\mathrm{a}} \Gamma_{\mathrm{a}} \Gamma_{z}\left(\tau_{+}+e^{\frac{z}{\ell}} \sigma_{-}\right)\right) .
\end{aligned}
$$

Again $\epsilon$ depends on four linearly independent spinors $\sigma_{ \pm}$and $\tau_{ \pm}$. As in the $A d S_{3}$ case substituting $^{4} \epsilon$ into (11.2), one finds that $\sigma_{ \pm}$and $\tau_{ \pm}$must satisfy (11.8). Repeating the argument we developed for the $A d S_{3}$ case, one concludes that $\epsilon$ does not factorize ${ }^{5}$ as in (11.1).

To illustrate the importance of keeping both components of $\sigma_{+}, \sigma_{+}^{+}$and $\sigma_{+}^{-}$, under the projection with $\Gamma_{z}$ for the correct counting of supersymmetries, let us consider the $A d S_{7} \times S^{4}$ background. One solves the algebraic KSEs, $\mathcal{A}^{(+)} \sigma_{+}=0$, after imposing the projection $^{6}$

$$
\Gamma_{z} \Gamma^{[4]} \sigma_{+}=\sigma_{+},
$$

where $\Gamma^{[4]}$ is the Clifford element associated with the volume form of $S^{4}$. This projection reduces the number of components of $\sigma_{+}$from 16 to 8 . Then the gravitino KSE can be solved without additional conditions. Using the intertwining Clifford algebra operators to find solitions to the KSEs for $\sigma_{-}$and $\tau_{ \pm}$, one concludes that $A d S_{7} \times S^{4}$ preserves 32 superymmetries as expected. However if one in addition imposes $\Gamma_{z} \sigma_{+}^{ \pm}= \pm \sigma_{+}^{ \pm}$in order for

\footnotetext{
${ }^{4}$ In the KSE (11.2) we use the spacetime gamma matrices. The same result holds if one uses the AdS gamma matrices after one decomposes $\sigma_{ \pm}$and $\tau_{ \pm}$in terms of AdS and transverse spinors.

${ }^{5}$ This questions the generality of several results that have been obtained in the literature using the factorization ansatz (11.1). How this additional assumption affects each computation is not apparent and the claims about the generality of several results have to be re-examined.

${ }^{6}$ The other projection $\Gamma_{z} \Gamma^{[4]} \sigma_{+}=-\sigma_{+}$can be treated in a similar way.
} 
the Killing spinor $\epsilon$ to satisfy (11.2), then one would incorrectly conclude that $A d S_{7} \times S^{4}$ preserves 16 supersymmetries.

We have demonstrated that the non-factorization of the Killing spinor as in (11.1) is crucial for the correct counting of supersymmetries of these backgrounds. On the other hand, the effect of the non-factorization of $\epsilon$ on the geometry of the transverse spaces $M^{11-n}$ is more subtle. A preliminary investigation [15] suggests that in some cases it has an effect but in some other cases it does not. Therefore it is required to investigate each case separately.

\section{$12 \mathbb{R}^{n-1,1}$ M-theory backgrounds}

As has been mentioned in the introduction the warped flat backgrounds $\mathbb{R}^{n-1,1} \times{ }_{w} M^{11-n}$ can be investigated in the context of $A d S_{n}$ backgrounds after taking the AdS radius $\ell$ to infinity. All our local computations are smooth in this limit. As a result, one can solve the KSEs, decompose the field equations and Bianchi identities as in the $A d S_{n}$ case and then take the limit $\ell \rightarrow \infty$ to derive the corresponding formulae for flat backgrounds. However in the limit, not all properties of $A d S_{n}$ backgrounds carry through. In particular the solutions to the KSEs will be re-examined as the process of integration yields powers of $\ell$ which alters the conclusions somewhat. To emphasize another difference, it is known for sometime that there are no smooth warped compactifications of 11-dimensional supergravity [29]. This is unless one appeals to M-theory and includes higher curvature corrections, due for example to anomaly cancellation, and/or additional brane charges. All these backgrounds are constructed starting from those of 11-dimensional supergravity and then appropriately correcting them. Since the 11-dimensional backgrounds are the starting point for such computations, we shall focus on these here.

\subsection{The warp factor is not nowhere vanishing}

One of the key properties of $A d S_{n}$ backgrounds is that the warp factor $A$ is nowhere vanishing. This is not the case for flat backgrounds. For flat backgrounds with non-trivial fluxes and under certain conditions, $A$ must always vanish somewhere. ${ }^{7}$ This follows from [29]. To see this focus on the $\mathbb{R}^{1,1}$ backgrounds and in particular in the field equation (3.6). After taking the limit $\ell \rightarrow \infty$ and assuming that the conditions on the fields and $M^{9}$ for the maximum principle to apply hold, one finds that the only solution to this equation is $A$ constant and $Y=X=0$, i.e. all the fluxes vanish, $F=0$. Therefore for compact $M^{9}$ and smooth fields, $A$ must vanish somewhere on $M^{9}$. A similar analysis can be done for the other flat backgrounds.

\subsection{Counting supersymmetries}

Because our global techniques do not straightforwardly apply to flat backgrounds, we shall focus on the counting of their supersymmetries based only on the local solution of the KSEs.

\footnotetext{
${ }^{7}$ From now on, we shall assume that $A$ is non-vanishing on an open subset of $M^{11-n}$ and restrict our analysis to that subset.
} 


\subsection{1 $\mathbb{R}^{1,1}$ backgrounds}

It is instructive to re-examine the integration of the KSEs in this case, in the limit that $\ell \rightarrow \infty, \Delta=0$. Using the expression for the Killing spinor in (2.23) and the integrability conditions (2.29) and (2.30), we find that

$$
\epsilon_{+}=\phi_{+}+u \Gamma_{+} \Theta_{-} \phi_{-}, \quad \epsilon_{-}=\phi_{-}+r \Gamma_{-} \Theta_{+} \phi_{+} .
$$

Therefore $\epsilon$ can depend on the lightcone coordinates $r, u$. Of course this dependence relies on $\phi_{ \pm} \notin \operatorname{Ker} \Theta_{ \pm}$. However, it should be stressed that $\phi_{ \pm}$satisfy automatically the integrability conditions (2.29) and (2.30) as a consequence of the remaining KSEs (3.9) on $M^{9}$, the field equations and Bianchi identities. Therefore, the dependence on the lightcone coordinates depends crucially on been able to find solutions of (3.9) which satisfy (2.29) and (2.30) but do not lie in the kernel of $\Theta_{ \pm}$.

The proof that we gave for $A d S_{2}$ backgrounds to preserve an even number of supersymmetries is not automatically valid for flat backgrounds. This is because it has been based on global considerations which are no longer valid for $\mathbb{R}^{1,1}$ solutions. Nevertheless, it is expected that some $\mathbb{R}^{1,1}$ backgrounds exhibit supersymmetry enhancement. Indeed suppose that $\phi_{-}$is a Killing spinor. Then we have shown by a local computation that $\phi_{+}=\Gamma_{+} \Theta_{-} \phi_{-}$is also a Killing spinor. Thus if $\phi_{-} \notin \operatorname{Ker} \Theta_{-}$, then such backgrounds will admit at least two supersymmetries.

\subsection{2 $\mathbb{R}^{2,1}$ backgrounds}

To determine the Killing spinors for this backgrounds, let us reexamine the solutions of the KSEs (2.23) along the additional $z$ direction of $\mathbb{R}^{2,1}$. The relevant equation is given in (5.10). To continue observe that the $\Xi^{( \pm)}$given in (5.11) satisfy

$$
\left(\Xi^{( \pm)}\right)^{2} \phi_{ \pm}=0
$$

in the limit $\ell \rightarrow \infty$ as a consequence of the integrability conditions (2.29) and (2.30). Thus the most general solution can be written as

$$
\phi_{ \pm}=\sigma_{ \pm}+z \Gamma_{z} A \Theta_{ \pm} \tau_{ \pm}, \quad \Xi^{( \pm)}\left(\sigma_{ \pm}-\tau_{ \pm}\right)=0
$$

where we have used that $\Xi^{( \pm)}=A \Gamma_{z} \Theta_{ \pm}$. Using again (12.2), the Killing spinors can be expressed as

$$
\epsilon_{+}=\sigma_{+}+u \Gamma_{+} \Theta_{-} \sigma_{-}+z \Gamma_{z} A \Theta_{+} \sigma_{+}, \quad \epsilon_{-}=\sigma_{-}+r \Gamma_{-} \Theta_{+} \sigma_{+}+z \Gamma_{z} A \Theta_{-} \sigma_{-} .
$$

Therefore the Killing spinors can depend on the coordinates ${ }^{8}$ of $\mathbb{R}^{2,1}$. But this depends crucially on $\sigma_{ \pm} \notin \operatorname{Ker} \Xi^{( \pm)}$even though they are in the kernel of $\left(\Xi^{( \pm)}\right)^{2}$ because of (12.2).

\footnotetext{
${ }^{8}$ As we shall see this is the case for the rest of the $\mathbb{R}^{n-1,1}$ backgrounds. It would be of interest to find solutions that exhibit this property, which is allowed from consideration of the KSEs, in order to test whether field equations and Bianchi identities impose additional conditions which remove this dependence.
} 
Furthermore a direct observation of the KSEs on $M^{8}(5.20)$ reveals that if $\sigma_{-}$is a solution, then

$$
\sigma_{+}=A^{-1} \Gamma_{+} \Gamma_{z} \sigma_{-}
$$

is also a solution, and vice versa if $\sigma_{+}$is a solution, then so is

$$
\sigma_{-}=A \Gamma_{-} \Gamma_{z} \sigma_{+} .
$$

Therefore all $\mathbb{R}^{2,1}$ backgrounds preserve an even number of supersymmetries confirming (1.3).

\subsection{3 $\mathbb{R}^{3,1}$ backgrounds}

As in the previous case, it is straightforward to observe that the solution to the KSEs along all $\mathbb{R}^{3,1}$ directions can be written as

$$
\begin{aligned}
& \epsilon_{+}=\sigma_{+}+u \Gamma_{+} \Theta_{-} \sigma_{-}+\left(z \Gamma_{z}+x \Gamma_{x}\right) A \Theta_{+} \sigma_{+}, \\
& \epsilon_{-}=\sigma_{-}+r \Gamma_{-} \Theta_{+} \sigma_{+}+\left(z \Gamma_{z}+x \Gamma_{x}\right) A \Theta_{-} \sigma_{-} .
\end{aligned}
$$

This is derived using $\left(\Xi^{( \pm)}\right)^{2} \phi_{ \pm}=0$ which in turn is implied from the integrability conditions (2.29) and (2.30) in the limit $\ell \rightarrow \infty$, and $\Xi^{( \pm)}=A \Gamma_{z} \Theta_{ \pm}$.

To verify (1.3), it remains to count the multiplicity of solutions to (7.11) in the limit $\ell \rightarrow \infty$. For this, if $\sigma_{-}$is a Killing spinor so is $\sigma_{+}=A^{-1} \Gamma_{+} \Gamma_{z} \sigma_{-}$and vice versa if $\sigma_{+}$is a Killing spinor so is $\sigma_{-}=A \Gamma_{-} \Gamma_{z} \sigma_{+}$. In addition, it follows from direct observation that if $\sigma_{+}$is a Killing spinor so is

$$
\sigma_{+}^{\prime}=\Gamma_{z x} \sigma_{+}
$$

and similarly for the $\sigma_{-}$. Therefore, $\mathbb{R}^{3,1}$ backgrounds preserve $4 k$ supersymmetries confirming (1.3).

\subsection{4 $\mathbb{R}^{n-1,1}, n>4$ backgrounds}

As in the previous cases, integrating the KSEs along the $\mathbb{R}^{n-1,1}$ directions yields

$$
\begin{aligned}
& \epsilon_{+}=\sigma_{+}+u \Gamma_{+} \Theta_{-} \sigma_{-}+\sum_{a} x^{a} \Gamma_{a} A \Theta_{+} \sigma_{+}, \\
& \epsilon_{-}=\sigma_{-}+r \Gamma_{-} \Theta_{+} \sigma_{+}+\sum_{a} x^{a} \Gamma_{a} A \Theta_{-} \sigma_{-} .
\end{aligned}
$$

where $\Gamma_{a}$ are in the frame basis in $\mathbb{R}^{n-1,1}$ and are transverse to the two lightcone directions + and - , and $x^{a}$ are the corresponding coordinates. The dependence of the Killing spinors on the coordinates of $\mathbb{R}^{n-1,1}$ depends on $\sigma_{ \pm} \notin \operatorname{Ker} \Xi^{( \pm)}$even though $\left(\Xi^{( \pm)}\right)^{2} \sigma_{ \pm}=0$ as a consequence of (2.29) and (2.30). Observe that in the limit $\ell \rightarrow \infty, \Xi^{( \pm)}=A \Gamma_{z} \Theta_{ \pm}$and that $\Theta_{+}=\Theta_{-}$.

To verify (1.3), it remains to count the multiplicity of solutions $\sigma_{ \pm}$of the remaining KSEs on $M^{11-n}$. As in the previous cases, if $\sigma_{-}$is a Killing spinor so is $\sigma_{+}=A^{-1} \Gamma_{+} \Gamma_{z} \sigma_{-}$ and vice versa if $\sigma_{+}$is a Killing spinor so is $\sigma_{-}=A \Gamma_{-} \Gamma_{z} \sigma_{+}$. 
Furthermore for $\mathbb{R}^{4,1}$ backgrounds it is easy to see that if $\sigma_{+}$is a Killing spinor, then $\Gamma_{a b} \sigma_{+}, a<b$, is also a Killing spinor, and similarly for the $\sigma_{-}$spinors. Therefore $\mathbb{R}^{4,1}$ backgrounds preserve $8 k$ supersymmetries confirming (1.3).

Next consider the $\mathbb{R}^{5,1}$. In this case, if $\sigma_{+}$is a Killing spinor, then $\Gamma_{a b} \sigma_{+}, a<b$ and

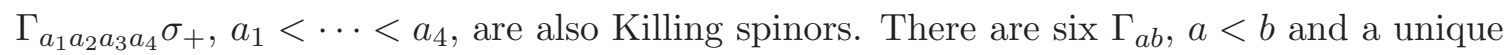
$\Gamma_{[4]}=\Gamma_{a_{1} a_{2} a_{3} a_{4}}, a_{1}<\cdots<a_{4}$, Clifford algebra operators. Moreover $\Gamma_{[4]}$ commutes with all the KSEs (9.10) and (9.11). Since $\Gamma_{[4]}^{2}=1, \sigma_{+}$can lie in one of the two eigenspaces of $\Gamma_{[4]}$. If this is the case, then only three of the $\Gamma_{a b} \sigma_{+}$are linearly independent. Thus $\mathbb{R}^{5,1}$ backgrounds preserve $8 k$ supersymmetries confirming (1.3).

To count the supersymmetries of $\mathbb{R}^{6,1}$ backgrounds observe that if $\sigma_{+}$is a Killing spinor so is $\Gamma_{a b} \sigma_{+}, a<b$ and $\Gamma_{a_{1} a_{2} a_{3} a_{4}} \sigma_{+}, a_{1}<\cdots<a_{4}$. There are ten $\Gamma_{a b}, a<b$ and five $\Gamma_{a_{1} a_{2} a_{3} a_{4}} \sigma_{+}, a_{1}<\cdots<a_{4}$, Clifford algebra operators. Furthermore all $\Gamma_{a_{1} a_{2} a_{3} a_{4}} \sigma_{+}$, $a_{1}<\cdots<a_{4}$ commute with the KSEs (9.10) and (9.11). Suppose we choose one such operator $\Gamma_{[4]}$. Since $\Gamma_{[4]}^{2}=1, \Gamma_{[4]}$ has two eigenspaces with eigenvalue \pm 1 . If $\sigma_{+}$is in one of the two eigenspaces, then there are only eight linearly independent spinors $\Gamma_{a b} \sigma_{+}, a<b$ and

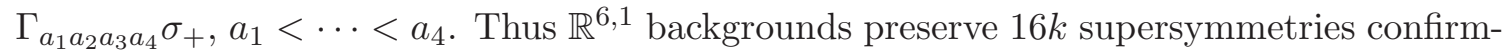
ing (1.3). Since there are no non-trivial $\mathbb{R}^{6,1}$ backgrounds with 32 supersymmetries [16], such solutions necessarily preserve 16 supersymmetries.

\section{Concluding remarks}

We have systematically described all warped AdS, $A d S_{n} \times{ }_{w} M^{11-n}$, and flat backgrounds, $\mathbb{R}^{n-1,1} \times_{w} M^{11-n}$, of $\mathrm{D}=11$ supergravity which preserve at least one supersymmetry. The novelty of our approach is that we have solved the KSEs of $D=11$ supergravity without making any assumptions on the form of the fields and Killing spinors. Integrating over all $A d S_{n}$ and flat directions, we have identified all the a priori fractions of supersymmetry preserved by these backgrounds. These results for AdS and flat backgrounds have been summarized in equations (1.1) and (1.3) in the introduction, respectively. Furthermore for AdS backgrounds that satisfy the requirements of maximum principle, we show that the Killing spinors can be identified with the zero modes of Dirac like operators on $M^{11-n}$. This identification is demonstrated via the proof of new Lichnerowicz type theorems for these Dirac like operators based on the identity (1.4). As a consequence we show that the number of Killing spinors of AdS backgrounds are given in terms of the dimension of the kernel of these Dirac like operators as in (1.2).

The general solution of the KSE of 11-dimensional supergravity for $A d S_{n} \times M^{11-n}$ backgrounds has allowed us to investigate whether the Killing spinors can be factorized as a product of a Killing spinor on $A d S_{n}$ and a Killing spinor on the transverse $M^{11-n}$. We have found that such a factorization does not occur. In particular we have demonstrated with an explicit example that assuming such a factorization gives an incorrect counting for the supersymmetries of AdS backgrounds.

The identification of the a priori fractions of supersymmetry preserved by AdS and flat backgrounds can be used to find all such solutions of 11-dimensional supergravity and Mtheory. This provides a systematic approach towards understanding all such backgrounds 
with applications in AdS/CFT and flux compactifications. There are already some preliminary results in this direction. In particular we find that under certain assumptions that are no $A d S_{6}$ backgrounds in $\mathrm{D}=11$ supergravity, however compare with [14] where the same results was proven assuming that the Killing spinors factorize. We also find that the geometry of $A d S_{2}$ backgrounds is less restricted than those investigated in [11, 12]. The proof of these results will be presented elsewhere [15].

Another aspect of our approach is the generalization of the classical Lichnerowicz theorem to non-metric connections. It is curious that the maximum principle which has been instrumental in the proof applies so widely in the context of supergravity. It is not known why this is so and it should be investigated further, as ultimately it may be related to supersymmetry.

\section{Acknowledgments}

JG is supported by the STFC grant, ST/1004874/1. JG would like to thank the Department of Mathematical Sciences, University of Liverpool for hospitality during which part of this work was completed. GP is partially supported by the STFC rolling grant ST/J002798/1.

\section{A Notation and conventions}

Our form conventions are as follows. Let $\omega$ be a k-form, then

$$
\omega=\frac{1}{k !} \omega_{i_{1} \ldots i_{k}} d x^{i_{1}} \wedge \cdots \wedge d x^{i_{k}}
$$

and

$$
d \omega=\frac{1}{k !} \partial_{i_{1}} \omega_{i_{2} \ldots i_{k+1}} d x^{i_{1}} \wedge \cdots \wedge d x^{i_{k+1}}
$$

leading to

$$
(d \omega)_{i_{1} \ldots i_{k+1}}=(k+1) \partial_{\left[i_{1}\right.} \omega_{\left.i_{2} \ldots i_{k+1}\right]} .
$$

Furthermore, we write

$$
\omega^{2}=\omega_{i_{1} \ldots i_{k}} \omega^{i_{1} \ldots i_{k}}, \quad \omega_{i_{1} i_{2}}^{2}=\omega_{i_{1} j_{1} \ldots j_{k-1}} \omega_{i_{2}}^{j_{1} \ldots j_{k-1}} .
$$

Given a volume form $d \mathrm{vol}=\frac{1}{n !} \epsilon_{i_{1} \ldots i_{n}} d x^{i_{1}} \wedge \cdots \wedge d x^{i_{n}}$, the Hodge dual of $\omega$ is defined as

$$
\chi \wedge * \omega=(\chi, \omega) d \mathrm{vol}
$$

where

$$
(\chi, \omega)=\frac{1}{k !} \chi_{i_{1} \ldots i_{k}} \omega^{i_{1} \ldots i_{k}}
$$

It is well-known that for every form $\omega$, one can define a Clifford algebra element $\psi$ given by

$$
\psi=\omega_{i_{1} \ldots i_{k}} \Gamma^{i_{1} \ldots i_{k}}
$$


where $\Gamma^{i}, i=1, \ldots n$, are the Dirac gamma matrices. In addition we introduce the notation

$$
\psi_{i_{1}}=\omega_{i_{1} i_{2} \ldots i_{k}} \Gamma^{i_{2} \ldots i_{k}}, \quad \not \omega_{i_{1}}=\Gamma_{i_{1}}{ }^{i_{2} \ldots i_{k+1}} \omega_{i_{2} \ldots i_{k+1}} .
$$

The rest of our spinor conventions can be found in [30, 31].

Open Access. This article is distributed under the terms of the Creative Commons Attribution License (CC-BY 4.0), which permits any use, distribution and reproduction in any medium, provided the original author(s) and source are credited.

\section{References}

[1] P.G.O. Freund and M.A. Rubin, Dynamics of Dimensional Reduction, Phys. Lett. B 97 (1980) 233 [INSPIRE].

[2] M.J. Duff, B.E.W. Nilsson and C.N. Pope, Kaluza-Klein Supergravity, Phys. Rept. 130 (1986) 1 [INSPIRE].

[3] M. Graña, Flux compactifications in string theory: A comprehensive review, Phys. Rept. 423 (2006) 91 [hep-th/0509003] [INSPIRE].

[4] O. Aharony, S.S. Gubser, J.M. Maldacena, H. Ooguri and Y. Oz, Large-N field theories, string theory and gravity, Phys. Rept. 323 (2000) 183 [hep-th/9905111] [INSPIRE].

[5] L. Castellani, L.J. Romans and N.P. Warner, A Classification of Compactifying Solutions for $d=11$ Supergravity, Nucl. Phys. B 241 (1984) 429 [INSPIRE].

[6] C.N. Pope and N.P. Warner, Two New Classes of Compactifications of $d=11$ Supergravity, Class. Quant. Grav. 2 (1985) L1 [inSPIRE].

[7] G.W. Gibbons and P.K. Townsend, Vacuum interpolation in supergravity via super p-branes, Phys. Rev. Lett. 71 (1993) 3754 [hep-th/9307049] [INSPIRE].

[8] B.S. Acharya, J.M. Figueroa-O'Farrill, C.M. Hull and B.J. Spence, Branes at conical singularities and holography, Adv. Theor. Math. Phys. 2 (1999) 1249 [hep-th/9808014] [INSPIRE].

[9] M. Cvetič, H. Lü, C.N. Pope and J.F. Vazquez-Poritz, AdS in warped space-times, Phys. Rev. D 62 (2000) 122003 [hep-th/0005246] [inSPIRE].

[10] J.P. Gauntlett, D. Martelli, J. Sparks and D. Waldram, Supersymmetric AdS $S_{5}$ solutions of M-theory, Class. Quant. Grav. 21 (2004) 4335 [hep-th/0402153] [INSPIRE].

[11] N. Kim and J.-D. Park, Comments on AdS $S_{2}$ solutions of $D=11$ supergravity, JHEP 09 (2006) 041 [hep-th/0607093] [INSPIRE].

[12] J.P. Gauntlett, N. Kim and D. Waldram, Supersymmetric $A d S_{3}, A d S_{2}$ and Bubble Solutions, JHEP 04 (2007) 005 [hep-th/0612253] [INSPIRE].

[13] M. Gabella, D. Martelli, A. Passias and J. Sparks, $\mathcal{N}=2$ supersymmetric AdS $S_{4}$ solutions of M-theory, Commun. Math. Phys. 325 (2014) 487 [arXiv:1207.3082] [INSPIRE].

[14] F. Apruzzi, M. Fazzi, A. Passias, D. Rosa and A. Tomasiello, AdS $S_{6}$ solutions of type-II supergravity, JHEP 11 (2014) 099 [arXiv:1406.0852] [INSPIRE].

[15] J. Gutowski and G. Papadopoulos, to appear. 
[16] J.M. Figueroa-O'Farrill and G. Papadopoulos, Maximally supersymmetric solutions of ten-dimensional and eleven-dimensional supergravities, JHEP 03 (2003) 048 [hep-th/0211089] [INSPIRE].

[17] U. Gran, J. Gutowski, G. Papadopoulos and D. Roest, $N=31, D=11$, JHEP 02 (2007) 043 [hep-th/0610331] [INSPIRE].

[18] U. Gran, J. Gutowski and G. Papadopoulos, M-theory backgrounds with 30 Killing spinors are maximally supersymmetric, JHEP 03 (2010) 112 [arXiv:1001.1103] [INSPIRE].

[19] J. Figueroa-O'Farrill and N. Hustler, The homogeneity theorem for supergravity backgrounds, JHEP 10 (2012) 014 [arXiv: 1208.0553] [INSPIRE].

[20] U. Gran, J. Gutowski and G. Papadopoulos, AdS backgrounds from black hole horizons, Class. Quant. Grav. 30 (2013) 055014 [arXiv:1110.0479] [INSPIRE].

[21] J. Gutowski and G. Papadopoulos, Static M-horizons, JHEP 01 (2012) 005 [arXiv:1106.3085] [INSPIRE].

[22] J. Gutowski and G. Papadopoulos, M-Horizons, JHEP 12 (2012) 100 [arXiv:1207.7086] [INSPIRE].

[23] J. Gutowski and G. Papadopoulos, Index theory and dynamical symmetry enhancement of M-horizons, JHEP 05 (2013) 088 [arXiv: 1303.0869] [INSPIRE].

[24] V. Moncrief and J. Isenberg, Symmetries of cosmological Cauchy horizons, Commun. Math. Phys. 89 (1983) 387 [InSPIRE].

[25] H. Friedrich, I. Racz and R.M. Wald, On the rigidity theorem for space-times with a stationary event horizon or a compact Cauchy horizon, Commun. Math. Phys. 204 (1999) 691 [gr-qc/9811021] [INSPIRE].

[26] E. Cremmer, B. Julia and J. Scherk, Supergravity Theory in Eleven-Dimensions, Phys. Lett. B 76 (1978) 409 [INSPIRE].

[27] M.F. Atiyah and I.M. Singer, The index of elliptic operators. I, Annals Math. 87 (1968) 484.

[28] T. Friedrich, Dirac Operators in Riemannian Geometry, Graduate Studies in Mathematics 25, AMS (2000).

[29] J.M. Maldacena and C. Núñez, Supergravity description of field theories on curved manifolds and a no go theorem, Int. J. Mod. Phys. A 16 (2001) 822 [hep-th/0007018] [InSPIRE].

[30] J. Gillard, U. Gran and G. Papadopoulos, The Spinorial geometry of supersymmetric backgrounds, Class. Quant. Grav. 22 (2005) 1033 [hep-th/0410155] [INSPIRE].

[31] U. Gran, G. Papadopoulos and D. Roest, Systematics of M-theory spinorial geometry, Class. Quant. Grav. 22 (2005) 2701 [hep-th/0503046] [INSPIRE]. 